\title{
Geometric Quantization on Kähler and Symplectic Manifolds
}

Xiaonan $\mathrm{Ma}^{*}$

\begin{abstract}
We explain various results on the asymptotic expansion of the Bergman kernel on Kähler manifolds and also on symplectic manifolds. We also review the "quantization commutes with reduction" phenomenon for a compact Lie group action, and its relation to the Bergman kernel.
\end{abstract}

Mathematics Subject Classification (2010). Primary 53D; Secondary 58J, 32A.

Keywords. Bergman kernel, Dirac operator, Geometric quantization, Index theorem.

\section{Introduction}

In the theory of quantization, one attempts to associate to a symplectic manifold $(X, \omega)$ a Hilbert space $H$ and a mapping from the space of functions on $X$ into the space of operators on $H$, and this in a canonical way. The mapping should give some reasonable relationship between the Poisson bracket on the function side and the commutator on the operator side. It is generally acknowledged that there is no canonical way to construct a quantization of $X$ without making use of certain additional structures.

In the theory of the geometric quantization of Kostant and Souriau, $(X, \omega)$ is assumed to be prequantizable, that is, there exists a prequantum line bundle $\left(L, h^{L}, \nabla^{L}\right)$ on $X$ (i.e., $\omega$ is the first Chern form of $L$ associated with the Hermitian connection $\nabla^{L}$ ). Given a compatible almost complex structure $J$ and a Riemannian metric $g^{T X}$, we can define canonically a Dirac operator $D^{L}$ acting on $\Omega^{0, \bullet}(X, L)$, the smooth $(0, \bullet)$-forms on $X$ with coefficients in $L$.

${ }^{*}$ We wish to express our thanks to Jean-Michel Bismut for discussions on various subjects and for his enlightened support. We would like to thank our collaborators Xianzhe Dai, Kefeng Liu, George Marinescu and Weiping Zhang for many helpful discussions. Thanks are due also to Institut Universitaire de France for support.

Université Paris Diderot - Paris 7, UFR de Mathématiques, Case 7012, Site Chevaleret, 75205 Paris Cedex 13, France. E-mail: ma@math.jussieu.fr. 
Assume that $X$ is compact. Following an observation by Bott, we take, as a quantization of $X, \operatorname{Ind}\left(D_{+}^{L}\right)=\operatorname{Ker}\left(D_{+}^{L}\right)-\operatorname{Coker}\left(D_{+}^{L}\right)$ of $D_{+}^{L}:=\left.D^{L}\right|_{\Omega^{0, \text { even }}}$, which is a formal difference of finite dimensional Hilbert spaces. The virtual dimension of $\operatorname{Ind}\left(D_{+}^{L}\right)$, which can be computed by the Atiyah-Singer index theorem, does not depend on the choice of the connection and of the metric on $L$.

For $p \gg 1, \operatorname{Ind}\left(D_{+}^{L^{p}}\right)=\operatorname{Ker}\left(D_{+}^{L^{p}}\right)$ is an ordinary finite dimensional Hilbert space. The Bergman kernel is defined as the integral kernel $P_{p}\left(x, x^{\prime}\right)$ associated with the orthogonal projection $P_{p}$ from $\Omega^{0, \bullet}\left(X, L^{p}\right)$ onto $\operatorname{Ker}\left(D^{L^{p}}\right)$. We will show that when $p \rightarrow+\infty$, the Bergman kernel $P_{p}\left(x, x^{\prime}\right)$ has an asymptotic expansion whose coefficients contain interesting geometric informations about $X$ and $L$. The kind of expansion obtained for the kernel $P_{p}\left(x, x^{\prime}\right)$ also characterizes the Berezin-Toeplitz operators. Their semi-classical limit provides a precise way to relate the classical and quantum observables.

Assume that a compact connected Lie group $G$ acts on $X$, and that the action lifts to $\left(L, h^{L}, \nabla^{L}\right)$. Then the quantization of $X$ is a $G$-virtual representation, and it is interesting to determine the multiplicity of the irreducible representations of $G$. The Guillemin-Sternberg conjecture "quantization commutes with reduction" gives a precise geometric answer to this problem by using the associated moment map. Here we explain the behavior of the $G$-invariant part of $P_{p}\left(x, x^{\prime}\right)$ as $p \rightarrow+\infty$, and we relate this behavior to the Guillemin-Sternberg conjecture.

New difficulties appear when the manifold $X$ is no longer supposed to be compact, since in this case $\operatorname{Ind}\left(D_{+}^{L}\right)$ is not well defined. In her ICM 2006 plenary lecture, Michèle Vergne proposed to replace $\operatorname{Ind}\left(D_{+}^{L}\right)$ by a certain transversal index introduced by Atiyah, under the natural hypothesis that the moment map is proper, and that the zero-set of the vector field induced by the moment map is compact. She conjectured that "quantization commutes with reduction" still holds in this case.

If $(X, \omega, J)$ is a compact Kähler manifold and if $L$ is holomorphic, then for $p \gg 1, \operatorname{Ker}\left(D^{L^{p}}\right)$ is the space of holomorphic sections $H^{0}\left(X, L^{p}\right)$ of $L^{p}$ on $X$. This leads to many applications of the asymptotic expansion of the Bergman kernel in Kähler geometry.

We refer the reader to our book with Marinescu [41] for a comprehensive study of the Bergman kernel and applications, and to the survey by Michèle Vergne [68] on the Guillemin-Sternberg conjecture. One can find more comments, references and motivations in [41] and [68].

This paper is organized as follows. The first two sections are based on our work with Dai, Liu and Marinescu, the last two sections are based on our work with Zhang. In Section 1, we review the definition of Bergman kernel and Berezin-Toeplitz quantization.

In Section 2, we discuss the asymptotic expansion of the Bergman kernel, and also Toeplitz operators. 
In Section 3, we examine the corresponding results when a compact Lie group $G$ acts on $X$ and the action lifts to $L$.

In Section 4, we outline Ma-Zhang's solution of the Vergne conjecture.

\section{Quantization on Symplectic Manifolds}

In Section 1.1, we review the basic definitions, and the spectral gap property of the Dirac operator. Then we explain the model example $\mathbb{C}^{n}$ in Section 1.2.

1.1. Dirac operators and quantization. Let $(X, \omega)$ be a compact symplectic manifold of real dimension $2 n$ with compatible almost complex structure $J$, i.e., $\omega(\cdot, J \cdot)>0, \omega(J \cdot, J \cdot)=\omega(\cdot, \cdot)$. We endow $X$ with a Riemannian metric $g^{T X}$ compatible with $J$, i.e., $g^{T X}(J \cdot, J \cdot)=g^{T X}(\cdot, \cdot)$. Let $\left(E, h^{E}\right)$ be a Hermitian vector bundle on $X$ with Hermitian connection $\nabla^{E}$ and curvature $R^{E}=\left(\nabla^{E}\right)^{2}$.

The almost complex structure $J$ induces a splitting of the complexification of the tangent bundle, $T X \otimes_{\mathbb{R}} \mathbb{C}=T^{(1,0)} X \oplus T^{(0,1)} X$, where $T^{(1,0)} X$ and $T^{(0,1)} X$ are the eigenbundles of $J$ corresponding to the eigenvalues $\sqrt{-1}$ and $-\sqrt{-1}$ respectively. Let $T^{*(0,1)} X$ be the dual space of $T^{(0,1)} X$. For any $v \in T^{(1,0)} X$, let $\bar{v}^{*} \in T^{*}(0,1) X$ be the metric dual of $v$, then

$$
\mathbf{c}(v)=\sqrt{2} \bar{v}^{*} \wedge, \quad \mathbf{c}(\bar{v})=-\sqrt{2} i_{\bar{v}},
$$

define the Clifford actions of $v, \bar{v}$ on $\Lambda^{0, \bullet}:=\Lambda^{\bullet}\left(T^{*(0,1)} X\right)$, where $\wedge$ and $i$ denote the exterior and interior multiplications respectively.

Consider the Levi-Civita connection $\nabla^{T X}$ of $\left(T X, g^{T X}\right)$ with associated curvature $R^{T X}$. Let $\nabla^{T^{(1,0)} X}$ be the connection on $T^{(1,0)} X$ induced by projecting $\nabla^{T X} ; \nabla^{T^{(1,0)} X}$ induces the connection $\nabla^{\text {det }}$ on $\operatorname{det}\left(T^{(1,0)} X\right)$. The Clifford connection $\nabla^{\mathrm{Cl}}$ on $\Lambda^{0, \bullet}$ is induced canonically by $\nabla^{T X}$ and $\nabla^{\operatorname{det}}$ (cf. [41, §1.3]). Finally, let $\nabla^{\Lambda^{0, \bullet}} \otimes E$ be the connection on $\Lambda^{0, \bullet} \otimes E$ induced by $\nabla^{\mathrm{Cl}}$ and $\nabla^{E}$.

Let $d v_{X}$ be the Riemannian volume form of $\left(T X, g^{T X}\right)$ and $\Omega^{0, \bullet}(X, E)$ be the space of smooth sections of $\Lambda^{0, \bullet} \otimes E$ endowed with the $L^{2}$-norm $\|\cdot\|_{L^{2}}$ induced by $h^{E}, g^{T X}$. Let $\left\{e_{j}\right\}_{j=1}^{2 n}$ be an orthonormal frame of $\left(T X, g^{T X}\right)$.

Definition 1.1. The spin $^{c}$ Dirac operator $D^{E}$ is defined by

$$
D^{E}:=\sum_{j} \mathbf{c}\left(e_{j}\right) \nabla_{e_{j}}^{\Lambda^{0, \bullet} \otimes E}: \Omega^{0, \bullet}(X, E) \longrightarrow \Omega^{0, \bullet}(X, E), \quad D_{ \pm}^{E}:=\left.D^{E}\right|_{\Omega^{0, \frac{\text { even }}{\text { odd }}}}
$$

The operator $D^{E}$ is a formally self-adjoint, first order elliptic differential operator on $\Omega^{0} \bullet(X, E)$, which interchanges $\Omega^{0, \text { even }}(X, E)$ and $\Omega^{0, \text { odd }}(X, E)$ (cf. [41, $\S 1.3])$. 
Thus $\operatorname{Ker}\left(D_{+}^{E}\right)$, Ker $\left(D_{-}^{E}\right)$ are finite dimensional Hilbert spaces and the quantization space of $E$ is defined as their formal difference

$$
Q(E):=\operatorname{Ind}\left(D_{+}^{E}\right):=\operatorname{Ker}\left(D_{+}^{E}\right)-\operatorname{Ker}\left(D_{-}^{E}\right) .
$$

The Atiyah-Singer index theorem [3, $\S 4.1]$, [41, Th. 1.3.9] allows us to compute the virtual dimension of $Q(E)$ by using characteristic numbers:

$$
\operatorname{dim} Q(E)=\int_{X} \operatorname{Td}\left(T^{(1,0)} X\right) \operatorname{ch}(E),
$$

where $\operatorname{ch}(\cdot), \operatorname{Td}(\cdot)$ are the Chern character and the Todd class of the corresponding complex vector bundles. In particular, the virtual dimension of $Q(E)$ does not depend on the choice of $J, g^{T X}$ or the metric and connection on $E$. If $\operatorname{Ker}\left(D_{-}^{E}\right)=0$, then the quantization space $Q(E)$ is an ordinary vector space.

We explain now the idea of the geometric quantization introduced by Kostant [33] and Souriau [62]. Let $\left(L, h^{L}\right)$ be a Hermitian line bundle over $X$ endowed with a Hermitian connection $\nabla^{L}$ with curvature $R^{L}=\left(\nabla^{L}\right)^{2}$. We assume that $\left(L, h^{L}, \nabla^{L}\right)$ satisfies the prequantization condition, that is

$$
\omega=\frac{\sqrt{-1}}{2 \pi} R^{L} .
$$

For $p \in \mathbb{N}$, we denote by $D^{L^{p} \otimes E}$ the Dirac operator associated to $L^{p} \otimes E$ with $L^{p}:=L^{\otimes p}$, and set

$$
E_{p}:=\Lambda^{0, \bullet} \otimes L^{p} \otimes E, \quad D_{p}:=D^{L^{p} \otimes E}, \quad D_{ \pm, p}:=\left.D_{p}\right|_{\Omega^{0}, \text { even }} .
$$

Let $L^{2}\left(X, E_{p}\right)$ be the $L^{2}$-completion of $\left(\Omega^{0} \bullet\left(X, L^{p} \otimes E\right),\|\cdot\|_{L^{2}}\right)$.

The following result is the starting point of the asymptotic expansion results for the Bergman kernel which we describe in the sequel. The proof is based on a direct application of the Lichnerowicz formula for $D_{p}^{2}$.

Theorem 1.2 (Ma-Marinescu [37, Th. 1.1,2.5], [41, Th. 1.5.5]). There exists $C>0$ such that for any $p \in \mathbb{N}$, the spectrum of $D_{p}^{2}$ satisfies

$$
\begin{aligned}
& \operatorname{Spec}\left(D_{p}^{2}\right) \subset\{0\} \cup\left[2 p \nu_{0}-C,+\infty[,\right. \\
& \operatorname{Ker}\left(D_{-, p}\right)=0 \quad \text { for } p \gg 1,
\end{aligned}
$$

where $\nu_{0}=\inf \left\{R_{x}^{L}(u, \bar{u}): u \in T_{x}^{(1,0)} X,|u|^{2}=1, x \in X\right\}>0$.

Thus for $p \gg 1, Q\left(L^{p} \otimes E\right)=\operatorname{Ker}\left(D_{p}^{2}\right)$ is an ordinary vector space and its dimension is a polynomial in $p$ of degree $n$ given by (1.4). The analogue of Theorem 1.2 in the holomorphic setting was first obtained by Bismut and Vasserot [8, Th. 1.1] by using Demailly's version of the Bochner-Kodaira-Nakano formula (cf. [41, Th. 1.4.12]). Formula (1.7b) was first established by Borthwick-Uribe [10, Th. 2.3] and Braverman [14, Th. 2.6] by using Melin's inequality. MathaiZhang [46, Th. 1.3] obtained a version of (1.7b) for the proper cocompact group action case by applying the method in [37]. 
Definition 1.3. The orthogonal projection $P_{p}: L^{2}\left(X, E_{p}\right) \longrightarrow \operatorname{Ker}\left(D_{p}\right)$ is called the Bergman projection. The Bergman kernel of $D_{p}$ is the smooth kernel $P_{p}\left(x, x^{\prime}\right) \in E_{p, x} \otimes E_{p, x^{\prime}}^{*},\left(x, x^{\prime} \in X\right)$, of $P_{p}$ with respect to $d v_{X}\left(x^{\prime}\right)$, i.e., for any $s \in L^{2}\left(X, E_{p}\right)$, we have

$$
\left(P_{p} s\right)(x)=\int_{X} P_{p}\left(x, x^{\prime}\right) s\left(x^{\prime}\right) d v_{X}\left(x^{\prime}\right) .
$$

For $f \in \mathscr{C}^{\infty}(X, \operatorname{End}(E))$, set

$$
T_{f, p}: L^{2}\left(X, E_{p}\right) \longrightarrow L^{2}\left(X, E_{p}\right), \quad T_{f, p}=P_{p} f P_{p} .
$$

Here the action of $f$ is the pointwise multiplication by $f$. The map which associates to $f \in \mathscr{C}^{\infty}(X, \operatorname{End}(E))$ the family of bounded operators $\left\{T_{f, p}\right\}_{p}$ on $L^{2}\left(X, E_{p}\right)$ is called the Berezin-Toeplitz quantization.

Definition 1.4. A Toeplitz operator is a sequence $\left\{T_{p}\right\}_{p \in \mathbb{N}}$ of linear operators $T_{p}: L^{2}\left(X, E_{p}\right) \longrightarrow L^{2}\left(X, E_{p}\right)$ satisfying $T_{p}=P_{p} T_{p} P_{p}$, such that there exists a sequence $g_{l} \in \mathscr{C}^{\infty}(X$, End $(E))$ such that for all $k \geqslant 0$, there exists $C_{k}>0$ with

$$
\left\|T_{p}-\sum_{l=0}^{k} T_{g_{l}, p} p^{-l}\right\| \leqslant C_{k} p^{-k-1} \quad \text { for any } p \in \mathbb{N}^{*},
$$

where $\|\cdot\|$ denotes the operator norm on the space of bounded operators. The section $g_{0}$ is called the principal symbol of $\left\{T_{p}\right\}$.

We express (1.10) symbolically by

$$
T_{p}=\sum_{l=0}^{k} T_{g_{l}, p} p^{-l}+\mathcal{O}\left(p^{-k-1}\right) .
$$

If (1.10) holds for any $k \in \mathbb{N}$, then we write (1.11) with $k=+\infty$.

The Poisson bracket $\{\cdot, \cdot\}$ on $(X, \omega)$ is defined as follows. For $f, g \in$ $\mathscr{C}^{\infty}(X)$, let $\xi_{f} \in \mathscr{C}^{\infty}(X, T X)$ be defined by $2 \pi i_{\xi_{f}} \omega=d f$. Then $\{f, g\}:=$ $\xi_{f}(d g)$.

In the spirit of the geometric quantization, $(X, \omega)$ represents the classical phase space and the Poisson algebra $\left(\mathscr{C}^{\infty}(X),\{\cdot\}\right)$ represents the classical observables, while $\operatorname{Ker}\left(D_{p}\right)$ is the quantum space and the linear operators on $\operatorname{Ker}\left(D_{p}\right)$ are the quantum observables. The process $p \rightarrow+\infty$ is called the semiclassical limit, which is a way to relate the classical and quantum observables.

1.2. Bergman kernel on $\mathbb{C}^{n}$. Let us consider the canonical real coordinates $\left(Z_{1}, \ldots, Z_{2 n}\right)$ on $\mathbb{R}^{2 n}$ and the complex coordinates $\left(z_{1}, \ldots, z_{n}\right)$ on $\mathbb{C}^{n}$. The two sets of coordinates are linked by the relation $z_{j}=Z_{2 j-1}+\sqrt{-1} Z_{2 j}$, $j=1, \ldots, n$. We consider the $L^{2}$-norm $\|\cdot\|_{L^{2}}=\left(\int_{\mathbb{R}^{2 n}}|\cdot|^{2} d Z\right)^{1 / 2}$ on the obvious $L^{2}$-space on $\mathbb{R}^{2 n}$, with $d Z=d Z_{1} \cdots d Z_{2 n}$ the Lebesgue measure. For $\alpha=\left(\alpha_{1}, \ldots, \alpha_{n}\right) \in \mathbb{N}^{n}, z \in \mathbb{C}^{n}$, put $z^{\alpha}=z_{1}^{\alpha_{1}} \cdots z_{n}^{\alpha_{n}}$. 
Let $L=\mathbb{C}$ be the trivial holomorphic line bundle on $\mathbb{C}^{n}$ with the canonical section $\mathbf{1}: \mathbb{C}^{n} \rightarrow L, z \mapsto(z, 1)$. Let $h^{L}$ be the metric on $L$ defined by

$$
|\mathbf{1}|_{h^{L}}(z):=e^{-\frac{1}{4} \sum_{j=1}^{n} a_{j}\left|z_{j}\right|^{2}}=\rho(Z) \quad \text { for } z \in \mathbb{C}^{n},
$$

with $a_{j}>0$ for $j \in\{1, \ldots, n\}$. The space of $L^{2}$-integrable holomorphic sections of $L$ with respect to $h^{L}$ and $d Z$ is the classical Segal-Bargmann space of $L^{2}$ integrable holomorphic functions with respect to the volume form $\rho d Z$. It is well-known that $\left\{z^{\beta}: \beta \in \mathbb{N}^{n}\right\}$ forms an orthogonal basis of this space.

To introduce the model operator $\mathscr{L}$ we set:

$$
b_{i}=-2 \frac{\partial}{\partial z_{i}}+\frac{1}{2} a_{i} \bar{z}_{i}, \quad b_{i}^{+}=2 \frac{\partial}{\partial \bar{z}_{i}}+\frac{1}{2} a_{i} z_{i}, \quad \mathscr{L}=\sum_{i} b_{i} b_{i}^{+} .
$$

We can interpret the operator $\mathscr{L}$ in terms of complex geometry. Let $\bar{\partial}^{L *}$ be the adjoint of the Dolbeault operator $\bar{\partial}^{L}$ on $\left(L, h^{L}\right)$ over $\left(\mathbb{C}^{n}, \frac{\sqrt{-1}}{2} \sum_{j} d z_{j} \wedge d \bar{z}_{j}\right)$. We have the isometry $\Omega^{0, \bullet}\left(\mathbb{C}^{n}, \mathbb{C}\right) \rightarrow \Omega^{0} \bullet\left(\mathbb{C}^{n}, L\right)$ given by $\alpha \mapsto \rho^{-1} \alpha$. If $\square^{L}=$ $\bar{\partial}^{L *} \bar{\partial}^{L}+\bar{\partial}^{L} \bar{\partial}^{L *}$ denotes the Kodaira Laplacian acting on $\Omega^{0} \bullet\left(\mathbb{C}^{n}, L\right)$, then $\rho \square^{L} \rho^{-1}: \Omega^{0, \bullet}\left(\mathbb{C}^{n}, \mathbb{C}\right) \rightarrow \Omega^{0, \bullet}\left(\mathbb{C}^{n}, \mathbb{C}\right)$ is given by $\frac{1}{2} \mathscr{L}+\sum_{j} a_{j} d \bar{z}^{j} \wedge i \frac{\partial}{\partial \bar{z}_{j}}$, and its restriction on functions is $\frac{1}{2} \mathscr{L}$.

The operator $\mathscr{L}$ is the complex analogue of the harmonic oscillator, the operators $b, b^{+}$are creation and annihilation operators respectively. Each eigenspace of $\mathscr{L}$ has infinite dimension, but we can still give an explicit description.

Theorem 1.5 (Ma-Marinescu [38, Th. 1.15], [41, Th.4.1.20]). The spectrum of $\mathscr{L}$ on $L^{2}\left(\mathbb{R}^{2 n}\right)$ is given by

$$
\operatorname{Spec}(\mathscr{L})=\left\{2 \sum_{i=1}^{n} \alpha_{i} a_{i}: \alpha=\left(\alpha_{1}, \cdots, \alpha_{n}\right) \in \mathbb{N}^{n}\right\}
$$

and an orthogonal basis of the eigenspace of $\lambda \in \operatorname{Spec}(\mathscr{L})$ is given by

$$
B_{\lambda}=\left\{b^{\alpha}\left(z^{\beta} \exp \left(-\frac{1}{4} \sum_{i} a_{i}\left|z_{i}\right|^{2}\right)\right): 2 \sum_{i} \alpha_{i} a_{i}=\lambda \text {, with } \alpha, \beta \in \mathbb{N}^{n}\right\}
$$

where $b^{\alpha}:=b_{1}^{\alpha_{1}} \cdots b_{n}^{\alpha_{n}}$. Moreover, $\cup_{\lambda}\left\{B_{\lambda}: \lambda \in \operatorname{Spec}(\mathscr{L})\right\}$ forms a complete orthogonal basis of $L^{2}\left(\mathbb{R}^{2 n}\right)$.

Let $\mathscr{P}\left(Z, Z^{\prime}\right)$ be the smooth kernel of $\mathscr{P}$, which is the orthogonal projection from $\left(L^{2}\left(\mathbb{R}^{2 n}\right),\|\cdot\|_{L^{2}}\right)$ onto $\operatorname{Ker}(\mathscr{L})$, with respect to $d Z^{\prime}$. Then $\mathscr{P}\left(Z, Z^{\prime}\right)$ is the classical Bergman kernel on $\mathbb{C}^{n}$ given by

$$
\mathscr{P}\left(Z, Z^{\prime}\right)=\prod_{i=1}^{n} \frac{a_{i}}{2 \pi} \exp \left(-\frac{1}{4} \sum_{i} a_{i}\left(\left|z_{i}\right|^{2}+\left|z_{i}^{\prime}\right|^{2}-2 z_{i} \bar{z}_{i}^{\prime}\right)\right) .
$$




\section{Asymptotic Expansion of Toeplitz Operators}

The starting point for our work on the asymptotic expansion of the Bergman kernel has been the heat equation proof by Bismut [6] of Demailly's holomorphic Morse inequalities [21]. For a unified treatment of these two questions, we refer to the book [41]. Here, we give various results on expansions of Bergman kernels, and also on Toeplitz operators.

This Section is organized as follows. In Section 2.1, we give the asymptotic expansion of the Bergman kernel.

In Section 2.2, we describe a characterization of the Toeplitz operators in terms of their asymptotic expansion.

In Section 2.3, we specify the results to the Kähler case.

We will use the notation and assumptions of Section 1.1.

2.1. Asymptotic expansion of Bergman kernel. Let $d^{X}\left(x, x^{\prime}\right)$ be the Riemannian distance between $x, x^{\prime} \in X$. Let $a^{X}$ be the injectivity radius of $\left(X, g^{T X}\right)$. We denote by $B^{X}(x, \varepsilon)$ and $B^{T_{x} X}(0, \varepsilon)$ the open balls in $X$ and $T_{x} X$ with centers $x$ and 0 and radius $\varepsilon$, respectively. Then the exponential $\operatorname{map} T_{x} X \ni Z \rightarrow \exp _{x}^{X}(Z) \in X$ is a diffeomorphism from $B^{T_{x} X}(0, \varepsilon)$ onto $B^{X}(x, \varepsilon)$ for $\varepsilon \leqslant a^{X}$. From now on, we identify $B^{T_{x} X}(0, \varepsilon)$ with $B^{X}(x, \varepsilon)$ via the exponential map for $\varepsilon \leqslant a^{X}$. When a function is calculated using normal coordinates based at $x$, we will add a subscript $x$.

We fix $x_{0} \in X$. For $Z \in B^{T_{x_{0}} X}(0, \varepsilon)$, we identify $E_{p, Z}$ with $E_{p, x_{0}}$ by parallel transport with respect to the connection $\nabla^{E_{p}}:=\nabla^{\Lambda^{0, \bullet} \otimes L^{p} \otimes E}$ along the curve $\gamma_{Z}:[0,1] \ni u \rightarrow u Z$.

Let $d v_{T X}$ be the Riemannian volume form on $\left(T_{x_{0}} X, g^{T_{x_{0}} X}\right)$. There exists a smooth positive function $\kappa_{x_{0}}$ on $B^{T_{x_{0}} X}(0, \varepsilon)$ defined by

$$
d v_{X}(Z)=\kappa_{x_{0}}(Z) d v_{T X}(Z), \quad \kappa_{x_{0}}(0)=1
$$

We will identify the 2-form $R^{L}$ with the Hermitian matrix $\dot{R}^{L} \in$ $\operatorname{End}\left(T^{(1,0)} X\right)$ such that for $W, Y \in T^{(1,0)} X, R^{L}(W, \bar{Y})=\left\langle\dot{R}^{L} W, \bar{Y}\right\rangle$. We choose an orthonormal basis $\left\{w_{i}\right\}_{i=1}^{n}$ of $T_{x_{0}}^{(1,0)} X$ such that

$$
\dot{R}^{L}\left(x_{0}\right)=\operatorname{diag}\left(a_{1}\left(x_{0}\right), \cdots, a_{n}\left(x_{0}\right)\right) \in \operatorname{End}\left(T_{x_{0}}^{(1,0)} X\right) \quad \text { with } a_{j}\left(x_{0}\right)>0 .
$$

Then $e_{2 j-1}=\frac{1}{\sqrt{2}}\left(w_{j}+\bar{w}_{j}\right)$ and $e_{2 j}=\frac{\sqrt{-1}}{\sqrt{2}}\left(w_{j}-\bar{w}_{j}\right), j=1, \ldots, n$, form an orthonormal basis of $T_{x_{0}} X$. We use the identification $\left(Z_{1}, \ldots, Z_{2 n}\right) \in \mathbb{R}^{2 n} \longrightarrow$ $\sum_{i} Z_{i} e_{i} \in T_{x_{0}} X$. In what follows, we also use the corresponding complex coordinates $z=\left(z_{1}, \ldots, z_{n}\right)$ on $\mathbb{C}^{n} \simeq \mathbb{R}^{2 n}$.

Let $\pi: T X \times_{X} T X \rightarrow X$ be the obvious projection. Let $\left\{\Theta_{p}\right\}_{p \in \mathbb{N}}$ be a sequence of linear operators $\Theta_{p}: L^{2}\left(X, E_{p}\right) \longrightarrow L^{2}\left(X, E_{p}\right)$ with smooth kernels $\Theta_{p}(x, y)$ with respect to $d v_{X}(y)$. In terms of our trivialization, $\Theta_{p}(x, y)$ induce smooth sections $\Theta_{p, x_{0}}\left(Z, Z^{\prime}\right)$ of $\pi^{*}\left(\operatorname{End}\left(\Lambda^{0, \bullet} \otimes E\right)\right)$ over $T X \times_{X} T X$, with $Z, Z^{\prime} \in T_{x_{0}} X$. Recall that $\mathscr{P}_{x_{0}}=\mathscr{P}$ was defined in (1.16). 
Notation 2.1. Let $\left\{Q_{r, x_{0}}\right\}_{0 \leqslant r \leqslant k, x_{0} \in X}$ be a family $Q_{r, x_{0}} \in \operatorname{End}\left(\Lambda^{0, \bullet} \otimes\right.$ $E)_{x_{0}}\left[Z, Z^{\prime}\right]$ of polynomials in $Z, Z^{\prime}$, smooth with respect to the parameter $x_{0} \in X$. We will write

$$
p^{-n} \Theta_{p, x_{0}}\left(Z, Z^{\prime}\right) \cong \sum_{r=0}^{k}\left(Q_{r, x_{0}} \mathscr{P}_{x_{0}}\right)\left(\sqrt{p} Z, \sqrt{p} Z^{\prime}\right) p^{-\frac{r}{2}}+\mathcal{O}\left(p^{-\frac{k+1}{2}}\right),
$$

if there exist $\left.\varepsilon^{\prime} \in\right] 0, a^{X}\left[, C_{0}>0\right.$ with the following property: for any $l \in \mathbb{N}$, there exist $C_{k, l}>0, M>0$ such that for any $x_{0} \in X, Z, Z^{\prime} \in T_{x_{0}} X,|Z|,\left|Z^{\prime}\right|<\varepsilon^{\prime}$ and $p \in \mathbb{N}^{*}$, the following estimate holds:

$$
\begin{aligned}
& \left|p^{-n} \Theta_{p, x_{0}}\left(Z, Z^{\prime}\right) \kappa_{x_{0}}^{\frac{1}{2}}(Z) \kappa_{x_{0}}^{\frac{1}{2}}\left(Z^{\prime}\right)-\sum_{r=0}^{k}\left(Q_{r, x_{0}} \mathscr{P}_{x_{0}}\right)\left(\sqrt{p} Z, \sqrt{p} Z^{\prime}\right) p^{-\frac{r}{2}}\right|_{\mathscr{C}^{l}(X)} \\
\leqslant & C_{k, l} p^{-\frac{k+1}{2}}\left(1+\sqrt{p}|Z|+\sqrt{p}\left|Z^{\prime}\right|\right)^{M} \exp \left(-\sqrt{C_{0} p}\left|Z-Z^{\prime}\right|\right)+\mathscr{O}\left(p^{-\infty}\right) .
\end{aligned}
$$

Here $|\cdot|_{\mathscr{C}^{l}(X)}$ is the $\mathscr{C}^{l}$ norm with respect to the parameter $x_{0} \in X$.

If $K \subset X \times X$ is compact, we will write that as $p \rightarrow+\infty, P_{p}\left(x, x^{\prime}\right)=$ $\mathscr{O}\left(p^{-\infty}\right)$ for $x, x^{\prime} \in K$ if for any $k, l \in \mathbb{N}$, the $\mathscr{C}^{l}$ norm of $P_{p}\left(x, x^{\prime}\right)$ for $x, x^{\prime} \in K$ with respect to the connections $\nabla^{L}, \nabla^{E}$ and the metrics $h^{L}, h^{E}, g^{T X}$ is dominated by $C p^{-k}$.

We denote by $I_{\mathbb{C} \otimes E}$ the projection from $\Lambda^{0, \bullet} \otimes E$ onto $\mathbb{C} \otimes E$ relative to the decomposition $\Lambda^{0, \bullet}=\mathbb{C} \oplus \Lambda^{0,>0}$.

We have the following full asymptotic expansion of the Bergman kernel.

Theorem 2.2 (Dai-Liu-Ma [20, Prop. 4.1 and Th.4.18'], [41, Th. 8.1.4]). For any $x_{0} \in X$ and $r \in \mathbb{N}$, there exist polynomials $J_{r, x_{0}}\left(Z, Z^{\prime}\right) \in \operatorname{End}\left(\Lambda^{0, \bullet} \otimes E\right)_{x_{0}}$ in $Z, Z^{\prime}$ with the same parity as $r$ and with $\operatorname{deg} J_{r, x_{0}} \leqslant 3 r$, whose coefficients are functions of the curvatures and their derivatives, such that for any $k \in \mathbb{N}$, in the sense of Notation 2.1,

$$
p^{-n} P_{p, x_{0}}\left(Z, Z^{\prime}\right) \cong \sum_{r=0}^{k}\left(J_{r, x_{0}} \mathscr{P}_{x_{0}}\right)\left(\sqrt{p} Z, \sqrt{p} Z^{\prime}\right) p^{-\frac{r}{2}}+\mathcal{O}\left(p^{-\frac{k+1}{2}}\right),
$$

with $J_{0, x_{0}}=I_{\mathbb{C} \otimes E}$. Moreover, for any $\varepsilon>0$, we have

$$
P_{p}\left(x, x^{\prime}\right)=\mathscr{O}\left(p^{-\infty}\right) \text { if } d^{X}\left(x, x^{\prime}\right) \geqslant \varepsilon .
$$

Idea of the proof. Using the spectral gap property in Theorem 1.2, and finite propagation speed of solutions of hyperbolic equations, we get (2.6). Also we can localize the asymptotics of $P_{p}\left(x_{0}, x^{\prime}\right)$ in the neighborhood of $x_{0}$. The second step consists in working on $\mathbb{R}^{2 n}$. To conclude the proof, we combine the spectral gap property, the rescaling of the coordinates and functional analytic techniques inspired by Bismut-Lebeau [7, §11]. 
By taking $\boldsymbol{b}_{r}\left(x_{0}\right)=\left(J_{2 r, x_{0}} \mathscr{P}_{x_{0}}\right)(0,0)$, we get from $(2.5)$ that for any $k, l \in \mathbb{N}$, there exists $C_{k, l}>0$ such that for any $p \in \mathbb{N}^{*}$,

$$
\left|P_{p}(x, x)-\sum_{r=0}^{k} \boldsymbol{b}_{r}(x) p^{n-r}\right|_{\mathscr{C} l(X)} \leqslant C_{k, l} p^{n-k-1} .
$$

We will give an algorithm to compute the coefficients $J_{r, x_{0}}$ in the expansion, by using a formal power series trick.

For $s \in \mathscr{C}^{\infty}\left(\mathbb{R}^{2 n},\left(\Lambda^{0, \bullet} \otimes E\right)_{x_{0}}\right), Z \in \mathbb{R}^{2 n},|Z| \leqslant \varepsilon$, and for $t=\frac{1}{\sqrt{p}}$, set

$$
\left(S_{t} s\right)(Z):=s(Z / t), \quad \mathscr{L}_{t}:=S_{t}^{-1} \kappa^{1 / 2} t^{2} D_{p}^{2} \kappa^{-1 / 2} S_{t} .
$$

By [20, Th. 4.6] (cf. [41, Th. 4.1.7]), there exist second order differential operators $\mathcal{O}_{r}$ such that for any $m \in \mathbb{N}$, we have an asymptotic expansion when $t \rightarrow 0$

$$
\mathscr{L}_{t}=\mathscr{L}_{0}+\sum_{r=1}^{m} t^{r} \mathcal{O}_{r}+\mathscr{O}\left(t^{m+1}\right), \quad \text { with } \mathscr{L}_{0}=\mathscr{L}+2 \sum_{j} a_{j} \bar{w}^{j} \wedge i_{\bar{w}_{j}} .
$$

Then $P^{N}=I_{\mathbb{C} \otimes E} \mathscr{P}$ is the orthogonal projection of $\left(L^{2}\left(\mathbb{R}^{2 n},\left(\Lambda^{0, \bullet} \otimes E\right)_{x_{0}}\right), \| \cdot\right.$ $\left.\|_{L^{2}}\right)$ onto $N=\operatorname{Ker}\left(\mathscr{L}_{0}\right)$. Set $P^{N^{\perp}}=\mathrm{Id}-P^{N}$. We define by recursion $f_{r}(\lambda) \in$ $\operatorname{End}\left(L^{2}\left(\mathbb{R}^{2 n},\left(\Lambda^{0, \bullet} \otimes E\right)_{x_{0}}\right)\right)$ by

$$
f_{0}(\lambda)=\left(\lambda-\mathscr{L}_{0}\right)^{-1}, \quad f_{r}(\lambda)=\left(\lambda-\mathscr{L}_{0}\right)^{-1} \sum_{j=1}^{r} \mathcal{O}_{j} f_{r-j}(\lambda) .
$$

Let $\delta$ be the counterclockwise oriented circle in $\mathbb{C}$ of center 0 and radius $\nu_{0} / 2$.

We denote by $\mathscr{F}_{r, x_{0}}$ the operator with smooth kernel

$$
\mathscr{F}_{r, x_{0}}\left(Z, Z^{\prime}\right)=J_{r, x_{0}}\left(Z, Z^{\prime}\right) \mathscr{P}\left(Z, Z^{\prime}\right)
$$

with respect to $d Z^{\prime}$. Then by $[38,(1.110)]$ (cf. also [41, (4.1.91)])

$$
\mathscr{F}_{r, x_{0}}=\frac{1}{2 \pi \sqrt{-1}} \int_{\delta} f_{r}(\lambda) d \lambda .
$$

By Theorem 1.5, (2.10), (2.12) and by the residue formula, we can express $\mathscr{F}_{r, x_{0}}$ in terms of $\mathscr{L}_{0}^{-1}, P^{N}, P^{N^{\perp}}, \mathcal{O}_{k}$ (with $\left.k \leqslant r\right)$. This gives a direct method to compute $\mathscr{F}_{r, x_{0}}$. In $[39, \S 2]$, we find an explicit computation for $\mathscr{F}_{2, x_{0}}$ when $\omega(\cdot, \cdot)=g^{T X}(J \cdot, \cdot)$ (i.e., $\dot{R}^{L}=2 \pi \mathrm{Id}$ ). We have in particular:

Theorem 2.3 (Ma-Marinescu [39, Th. 2.1]). If $\omega(\cdot, \cdot)=g^{T X}(J \cdot, \cdot)$, we have

$$
\left.\operatorname{Tr}\right|_{\Lambda\left(T^{*(0,1) X}\right)}\left[\boldsymbol{b}_{1}(x)\right]=\frac{1}{8 \pi}\left[r^{X}+\frac{1}{4}\left|\nabla^{X} J\right|^{2}+4 \sum_{j} R^{E}\left(w_{j}, \bar{w}_{j}\right)\right] .
$$


Here $\nabla^{X} J$ is the covariant derivative of $J$ with respect to $\nabla^{T X}$, and $r^{X}$ is the scalar curvature of $\left(X, g^{T X}\right)$. In Donaldson [22], the term $r^{X}+\frac{1}{4}\left|\nabla^{X} J\right|^{2}$ in (2.13) is called the Hermitian scalar curvature. It is a natural substitute for the Riemannian scalar curvature in the almost-Kähler case. It was used by Donaldson to define the moment map on the space of compatible almostcomplex structures.

Ma-Zhang [44] obtained a family version of Theorem 2.2.

2.2. Asymptotic expansion of Toeplitz operators. Here is a useful characterization of the Toeplitz operators in terms of their kernel.

Theorem 2.4. (Ma-Marinescu $[40, \quad$ Th. 4.9, Rem.4.10], [41, Lemmas 7.2.2, 7.2.4, Th.7.3.1]) Let $\left\{T_{p}: L^{2}\left(X, E_{p}\right) \longrightarrow L^{2}\left(X, E_{p}\right)\right\}$ be a family of bounded linear operators. Then $\left\{T_{p}\right\}$ is a Toeplitz operator if and only if it satisfies the following three conditions:

(i) For any $p \in \mathbb{N}, P_{p} T_{p} P_{p}=T_{p}$.

(ii) For any $\varepsilon_{0}>0, T_{p}\left(x, x^{\prime}\right)=\mathscr{O}\left(p^{-\infty}\right)$ if $d^{X}\left(x, x^{\prime}\right) \geqslant \varepsilon_{0}$.

(iii) There exists a family of polynomials $\left\{Q_{r, x_{0}} \in \operatorname{End}\left(\Lambda^{0, \bullet} \otimes\right.\right.$ $\left.E)_{x_{0}}\left[Z, Z^{\prime}\right]\right\}_{x_{0} \in X}$ which has the same parity as $r$, such that for any $k \in \mathbb{N}$, we have in the sense of (2.3) and (2.4),

$$
p^{-n} T_{p, x_{0}}\left(Z, Z^{\prime}\right) \cong \sum_{r=0}^{k}\left(Q_{r, x_{0}} \mathscr{P}_{x_{0}}\right)\left(\sqrt{p} Z, \sqrt{p} Z^{\prime}\right) p^{-\frac{r}{2}}+\mathcal{O}\left(p^{-\frac{k+1}{2}}\right) .
$$

In this case, its principal symbol is $g_{0}\left(x_{0}\right)=\left.Q_{0, x_{0}}(0,0)\right|_{\mathbb{C} \otimes E} \in \operatorname{End}\left(E_{x_{0}}\right)$.

Remark 2.5. For $f \in \mathscr{C}^{\infty}(X, \operatorname{End}(E))$, conditions (i), (ii), (iii) of Theorem 2.4 for $\left\{T_{f, p}\right\}$ are consequences of Theorem 2.2 and of the Taylor expansion of $f$ at $x_{0}$. The coefficients $Q_{r, x_{0}}$ in (2.14) corresponding to the Toeplitz operator $\left\{T_{f, p}\right\}$ are denoted by $Q_{r, x_{0}}(f)$, and $Q_{0, x_{0}}(f)=f\left(x_{0}\right) I_{\mathbb{C} \otimes E}$.

By taking $\boldsymbol{b}_{r, f}\left(x_{0}\right)=\left(Q_{2 r, x_{0}}(f) \mathscr{P}_{x_{0}}\right)(0,0)$, we get from (2.14) that for any $k, l \in \mathbb{N}$, there exists $C_{k, l}>0$ such that for any $p \in \mathbb{N}^{*}$, we have

$$
\left|T_{f, p}(x, x)-\sum_{r=0}^{k} \boldsymbol{b}_{r, f}(x) p^{n-r}\right|_{\mathscr{C}^{l}(X)} \leqslant C_{k, l} p^{n-k-1} .
$$

In [40, (4.15)] (cf. also [41, (7.2.16)]), we find a precise formula for $Q_{r, x_{0}}(f)$ by using the Taylor expansion of $f$ at $x_{0}, J_{j, x_{0}}(j \leqslant r)$ and $\mathscr{P}_{x_{0}}$ in $(2.5)$, from which the computation $\boldsymbol{b}_{r, f}\left(x_{0}\right)$ can be derived.

Theorem 2.6 (Ma-Marinescu [40, Th. 1.1], [41, Th.7.4.1]). The product of the Toeplitz operators $T_{f, p}$ and $T_{g, p}$, with $f, g \in \mathscr{C}^{\infty}(X, \operatorname{End}(E))$, is a Toeplitz 
operator, i.e., it admits the asymptotic expansion in the sense of (1.11):

$$
T_{f, p} T_{g, p}=\sum_{r=0}^{\infty} p^{-r} T_{C_{r}(f, g), p}+\mathcal{O}\left(p^{-\infty}\right)
$$

where $C_{r}$ are bidifferential operators, $C_{0}(f, g)=f g$ and $C_{r}(f, g) \in$ $\mathscr{C}^{\infty}(X, \operatorname{End}(E))$.

If $f, g \in\left(\mathscr{C}^{\infty}(X),\{\cdot, \cdot\}\right)$ with the Poisson bracket defined in Section 1.1, we have

$$
\left[T_{f, p}, T_{g, p}\right]=\frac{\sqrt{-1}}{p} T_{\{f, g\}, p}+\mathcal{O}\left(p^{-2}\right) .
$$

Theorem 2.6 implies that the set of Toeplitz operators is closed under the composition of operators, and so it forms an associative algebra.

For $E=\mathbb{C}$, Theorem 2.6 shows that we can associate to $f, g \in \mathscr{C}^{\infty}(X)$ a formal power series $\sum_{l=0}^{\infty} \hbar^{l} C_{l}(f, g) \in \mathscr{C}^{\infty}(X)[[\hbar]]$, where $C_{l}$ are bidifferential operators. Therefore, we have constructed in a canonical way an associative star-product $f * g=\sum_{l=0}^{\infty} \hbar^{l} C_{l}(f, g)$, called the Berezin-Toeplitz star-product. Note that the existence of formal star product on symplectic manifolds was established by De Wilde and Lecomte in 1983. We refer to Fedosov's book [24] for more information on the theory of deformation quantization. In Theorem 2.6, we gave a geometric realization of the associative star-product.

2.3. The Kähler case. In this subsection, we assume that $(X, \omega, J)$ is a compact Kähler manifold, $\left(L, h^{L}\right)$ is a holomorphic Hermitian line bundle with Chern connection $\nabla^{L}$ verifying $(1.5)$, and $\left(E, h^{E}\right)$ is a holomorphic Hermitian vector bundle with Chern connection $\nabla^{E}$. We assume also that $\omega=\frac{\sqrt{-1}}{2 \pi} R^{L}$ is the Kähler form of $\left(X, g^{T X}\right)$. Let $\bar{\partial}^{L^{p} \otimes E, *}$ be the adjoint of the Dolbeault operator $\bar{\partial}^{L^{p} \otimes E}$ on $\Omega^{0, \bullet}\left(X, L^{p} \otimes E\right)$. In this case, $D_{p}$ in (1.6) is given by

$$
D_{p}=\sqrt{2}\left(\bar{\partial}^{L^{p} \otimes E}+\bar{\partial}^{L^{p} \otimes E, *}\right) .
$$

Thus $D_{p}^{2}$ preserves the $\mathbb{Z}$-grading on $\Omega^{0, \bullet}\left(X, L^{p} \otimes E\right)$. By Hodge theory and the Kodaira vanishing theorem, we have

$$
\operatorname{Ker}\left(D_{p}\right)=H^{0}\left(X, L^{p} \otimes E\right) \quad \text { for } p \gg 1 .
$$

The Bergman projection $P_{p}$ reduces to a projection from $\mathscr{C}^{\infty}\left(X, L^{p} \otimes E\right)$ onto $H^{0}\left(X, L^{p} \otimes E\right)$, a Toeplitz operator $\left\{T_{p}\right\}$ is now a sequence of linear operators acting on $\mathscr{C}^{\infty}\left(X, L^{p} \otimes E\right)$. Thus we don't need to introduce differential forms, and we can work on $\mathscr{C}^{\infty}\left(X, L^{p} \otimes E\right)$. In this situation, $J_{r, x_{0}}, \boldsymbol{b}_{r}\left(x_{0}\right)$, $Q_{r, x_{0}}(f), \boldsymbol{b}_{r, f}\left(x_{0}\right)$ introduced in (2.5), (2.7), Remark 2.5 and (2.15) take values in $\operatorname{End}(E)_{x_{0}}$.

Let $\mathbb{P}\left(H^{0}\left(X, L^{p}\right)^{*}\right)$ be the projective space associated to the dual of $H^{0}\left(X, L^{p}\right)$, and let $\omega_{F S}$ be the Fubini-Study $(1,1)$-form. The Kodaira map 
$\phi_{p}: X \longrightarrow \mathbb{P}\left(H^{0}\left(X, L^{p}\right)^{*}\right)$ is defined by $\phi_{p}(x)=\left\{H^{0}\left(X, L^{p}\right) \ni s \rightarrow s(x) \in L_{x}^{p}\right\}$ for $x \in X$. The Kodaira embedding theorem asserts that for $p \gg 1, \phi_{p}$ is a holomorphic embedding and $\phi_{p}^{*} \mathscr{O}(1)=L^{p}$. Let $h_{p}^{\phi_{p}^{*} \mathscr{O}(1)}$ be the metric on $\phi_{p}^{*} \mathscr{O}(1)$ induced by the metric $h^{\mathscr{O}(1)}$ on $\mathscr{O}(1)$. Then for $E=\mathbb{C}$, we have (cf. [41, Th. $5.1 .3])$

$$
h^{\phi_{p}^{*} \mathscr{O}(1)}(x)=P_{p}(x, x)^{-1} h^{L^{p}}(x) .
$$

The question of the convergence as $p \rightarrow+\infty$ of $\frac{1}{p} \phi_{p}^{*}\left(\omega_{F S}\right)$ was raised by Yau $[71, \S 6.1]$. By $(2.7)$ for $E=\mathbb{C}$, and $(2.20)$, as $p \rightarrow+\infty, \frac{1}{p} \phi_{p}^{*}\left(\omega_{F S}\right)$ converges to $\omega$ in the $\mathscr{C}^{\infty}$ topology: for any $l \geqslant 0$, there exists $C_{l}>0$ such that

$$
\left|\frac{1}{p} \phi_{p}^{*}\left(\omega_{F S}\right)-\omega\right|_{\mathscr{C}^{l}(X)} \leqslant C_{l} / p^{2} .
$$

When $l=2$, the estimate of the type (2.21) was obtained by Tian [64] with $p^{2}$ replaced by $\sqrt{p}$, by using the Bergman kernel on the diagonal, $P_{p}(x, x)$. Ruan [59] obtained (2.21) with $p$ instead of $p^{2}$. Bouche [11] proved that $\lim _{p \rightarrow+\infty} p^{-n} P_{p}(x, x)=1$ in the $\mathscr{C}^{0}$ topology. The expansion (2.7) was first established by Catlin [17] and Zelditch [72].

$\mathrm{Lu}$ [36] calculated more coefficients $\boldsymbol{b}_{r}$ via $R^{T X}$. Let $\operatorname{Ric}=\operatorname{Ric}_{g}(J \cdot, \cdot)$ be the $(1,1)$-form associated to the Ricci curvature $\operatorname{Ric}_{g}$ of $g^{T X}$. Let $\Delta$ be the positive Laplacian acting on functions on $X$; set $\left|R^{T X}\right|^{2}=\sum_{i j k l} \mid\left\langle R^{T X}\left(w_{i}, \bar{w}_{j}\right) w_{k}\right.$, $\left.\bar{w}_{j}\right\rangle\left.\right|^{2}$.

Theorem 2.7 (Lu [36, Th. 1.1]). When $E=\mathbb{C}$, we have

$$
\boldsymbol{b}_{1}=\frac{r^{X}}{8 \pi}, \quad \pi^{2} \boldsymbol{b}_{2}=-\frac{\Delta r^{X}}{48}+\frac{1}{96}\left|R^{T X}\right|^{2}-\frac{1}{24}|\operatorname{Ric}|^{2}+\frac{1}{128}\left(r^{X}\right)^{2} .
$$

Wang [70] also computed $\boldsymbol{b}_{1}$ in (2.7) for general $E$. When $E=\mathbb{C}$, the existence of an asymptotic expansion similar to $(2.5)$ for $|Z|,\left|Z^{\prime}\right| \leqslant C / \sqrt{p}$ was also obtained in [61, Th. 1]. For other versions of the asymptotic expansion see [17], [31], [18], [4]. The main tool in [17], [72], [18], [31], and [61] is the Boutet de Monvel-Sjöstrand parametrix for the Szegö kernel [13], [25]. The coefficients were computed in [64], [36], [70] by constructing appropriate peak sections, using Hörmander's $L^{2} \bar{\partial}$-method.

If $E=\mathbb{C}$, the existence of the expansion (2.16) was first established by Bordemann, Meinrenken and Schlichenmaier [9], Schlichenmaier [60], [31]. They used the theory of Toeplitz structures of Boutet de Monvel and Guillemin [12].

Lu's computation for $\boldsymbol{b}_{1}$ plays an important role in Donaldson's work [23] on Kähler metrics with constant scalar curvature. We refer to [5], [41] for further information. In [42], we computed the coefficients $\boldsymbol{b}_{1, f}, \boldsymbol{b}_{2, f}, C_{1}(f, g), C_{2}(f, g)$ from $(2.15),(2.16)$. These computations are also relevant in Kähler geometry (cf. [26], [27], [35]). 
Theorem 2.8 (Ma-Marinescu [42]). If $E=\mathbb{C}$, for any $f \in \mathscr{C}^{\infty}(X)$, we have:

$$
\begin{aligned}
& \boldsymbol{b}_{0, f}=f, \quad \boldsymbol{b}_{1, f}=\frac{r^{X}}{8 \pi} f-\frac{1}{4 \pi} \Delta f \\
& \boldsymbol{b}_{2, f}=\boldsymbol{b}_{2} f+\frac{1}{32 \pi^{2}} \Delta^{2} f-\frac{1}{32 \pi^{2}} r^{X} \Delta f-\frac{\sqrt{-1}}{8 \pi^{2}}\langle\text { Ric }, \partial \bar{\partial} f\rangle .
\end{aligned}
$$

\section{Quantization and Symplectic Reduction}

We explain briefly the Guillemin-Sternberg conjecture in Section 3.1, then we review the asymptotic expansion of the $G$-invariant part of the Bergman kernel in Section 3.2, and we specialize the results in the Kähler case in Section 3.3. In particular, we show how to obtain the scalar curvature on the reduction from the $G$-invariant Bergman kernel on the total space, and we compare the metrics on the two sides of the "quantization commutes with reduction".

We use the same notation and assumptions as in Section 1.1.

3.1. Quantization commutes with reduction. Recall that $(X, \omega, J)$ is a compact symplectic manifold of real dimension $2 n$ with compatible almost complex structure $J$, and $\left(L, h^{L}, \nabla^{L}\right)$ is a prequantum line bundle on $X$ (cf. (1.5)).

Let $G$ be a compact connected Lie group of dimension $n_{0}$ with Lie algebra $\mathfrak{g}$. We assume that $G$ acts on the left on $X$ and that this action lifts to $L$. Moreover, we assume that $G$ preserves $g^{T X}, J, h^{L}$ and $\nabla^{L}$.

The $G$-action commutes with the Dirac operator $D^{L}$, and $\operatorname{Ker}\left(D_{ \pm}^{L}\right)$ are finite dimensional $G$-representations. The quantization space $Q(L)$ of $L$ (cf. (1.3)) is an element in the representation $\operatorname{ring} R(G)$ of $G$.

For $K \in \mathfrak{g}$, let $K^{X}$ be the vector field on $X$ generated by $K$, and let $L_{K}$ be the corresponding Lie derivative. Let $\Lambda_{+}^{*} \subset \mathfrak{g}^{*}$ be the set of dominant weights, and let $V_{\gamma}^{G}$ be the irreducible representation of $G$ with highest weight $\gamma \in \Lambda_{+}^{*}$. Let $Q(L)^{\gamma} \in \mathbb{Z}$ be the multiplicity of $V_{\gamma}^{G}$ in $Q(L)$. Then we have

$$
Q(L)=\bigoplus_{\gamma \in \Lambda_{+}^{*}} Q(L)^{\gamma} \cdot V_{\gamma}^{G} \in R(G),
$$

and there are only finitely many $\gamma \in \Lambda_{+}^{*}$ such that $Q(L)^{\gamma} \neq 0$.

It is not easy to read off $Q(L)^{\gamma}$ directly from the Atiyah-Bott-Segal-Singer equivariant index theorem for its character. Guillemin and Sternberg [29] suggested a geometric way to compute $Q(L)^{\gamma}$, by using the associated moment map.

Definition 3.1. The moment map $\mu: X \rightarrow \mathrm{g}^{*}$ is defined by the Kostant formula [33],

$$
2 \sqrt{-1} \pi \mu(K)=\nabla_{K^{x}}^{L}-L_{K}, \quad \text { for } K \in \mathfrak{g} .
$$

Then $\mu$ is $G$-equivariant and one has $i_{K} x \omega=d \mu(K)$. 
For a regular value $\nu \in \mathfrak{g}^{*}$ of $\mu$, the Marsden-Weinstein symplectic reduction $X_{\nu}:=\mu^{-1}(G \cdot \nu) / G$ is a compact symplectic orbifold with the symplectic form $\omega_{\nu}$ induced by $\omega$. Moreover, $L$ (resp. $J$ ) induces a prequantum line bundle $L_{\nu}$ (resp. an almost complex structure $J_{\nu}$ ) over $\left(X_{\nu}, \omega_{\nu}\right)$. One can then construct the associated $\operatorname{spin}^{c}$ Dirac operator (twisted by $\left.L_{\nu}\right), D_{+}^{L_{\nu}}$ on $X_{\nu}$, of which the index $Q\left(L_{\nu}\right) \in \mathbb{Z}$ (identified as the virtual dimension of $Q\left(L_{\nu}\right)$ in (1.3)).

If $\gamma \in \Lambda_{+}^{*}$ is not a regular value of $\mu$, then by [49] (cf. [54, $\left.\S 7.4\right],[43, \S 3.5]$ for a standard perturbation definition), $Q\left(L_{\gamma}\right)$ is still well defined. Now we can state:

Guillemin-Sternberg conjecture: For any $\gamma \in \Lambda_{+}^{*}$,

$$
Q(L)^{\gamma}=Q\left(L_{\gamma}\right)
$$

By the classical shifting trick (i.e., by working on $X \times \mathcal{O}_{\gamma}$, where $\mathcal{O}_{\gamma}=G \cdot \gamma$ is the orbit of the co-adjoint action of $G$ on $\mathfrak{g}^{*}$ ), we only need to prove (3.3) for $\gamma=0$.

This conjecture was proved by Meinrenken [47] and Vergne [67] when $G$ is abelian; by Meinrenken [48], Meinrenken-Sjamaar[49] for non-abelian groups $G$, by using the technique of symplectic cut of Lerman [34].

Tian and Zhang [65] gave an analytic proof of the Guillemin-Sternberg conjecture, using a deformation of the Dirac operator, which is associated with the function $|\mu|^{2}$. Their approach works for a general vector bundle $E$ satisfying certain positivity conditions [65, (4.2)] (used afterwards by Paradan [54, p. 445] and Teleman [63, p.6]), and also for manifolds with boundary [66]. Paradan [54] developed later a $K$-theoretic approach by making use of the theory of transversally elliptic operators. See [68] for a survey and complete references on this subject.

3.2. Berezin-Toeplitz quantization and reduction. We use the same notation and assumptions as in Sections 1.1 and 3.1. We assume also that the $G$-action lifts on $E$ and preserves $h^{E}$ and $\nabla^{E}$.

Then $G$-action commutes with the Dirac operator $D_{p}$ in (1.6). Let $\operatorname{Ker}\left(D_{p}\right)^{G}$ be the $G$-trivial component of $\operatorname{Ker}\left(D_{p}\right)$. Let $P_{p}^{G}$ be the orthogonal projection from $\mathscr{C}^{\infty}\left(X, E_{p}\right)$ onto $\operatorname{Ker}\left(D_{p}\right)^{G}$. The $G$-invariant Bergman kernel is the $\mathscr{C}^{\infty}$ kernel $P_{p}^{G}\left(x, x^{\prime}\right),\left(x, x^{\prime} \in X\right)$ of $P_{p}^{G}$ associated to $d v_{X}\left(x^{\prime}\right)$.

Assume for simplicity that $G$ acts freely on $\mu^{-1}(0)$, and $g^{T X}(\cdot, \cdot)=\omega(\cdot, J \cdot)$. We will denote by $X_{G}=\mu^{-1}(0) / G$, and we add a subscript $G$ to denote the objects on $X_{G}$ induced by the corresponding objects on $X$.

By a result of Tian and Zhang [65, Th. 0.2], and (1.7b), we have

$$
\operatorname{dim} \operatorname{Ker}\left(D_{p}\right)^{G}=\operatorname{dim} \operatorname{Ker}\left(D_{G, p}\right) \quad \text { for } p \gg 1 .
$$

We will describe how $P_{p}^{G}\left(x, x^{\prime}\right)$ "concentrates" on the Bergman kernel $P_{G, p}\left(x_{0}, x_{0}^{\prime}\right)$ on $X_{G}$, when $p \rightarrow+\infty$. 
Theorem 3.2 (Ma-Zhang [43, Th. 0.1]). For any open G-neighborhood $U$ of $\mu^{-1}(0)$ and any $\varepsilon_{0}>0$, we have

$$
P_{p}^{G}\left(x, x^{\prime}\right)=\mathscr{O}\left(p^{-\infty}\right) \text { if }\left(x, x^{\prime}\right) \notin U \times U \text { or if } d^{X}\left(G x, x^{\prime}\right) \geqslant \varepsilon_{0} .
$$

Let $U$ be an open $G$-neighborhood of $\mu^{-1}(0)$ such that $G$ acts freely on $U$. For any $G$-equivariant vector bundle with connection $\left(F, \nabla^{F}\right)$ on $U$, we denote by $\left(F_{B}, \nabla^{F_{B}}\right)$ the bundle on $B:=U / G$ induced by $G$-invariant sections of $F$ on $U$.

For $x \in U$ denote by $\operatorname{vol}(G x)$ the volume of the orbit $G x$ equipped with the metric induced by $g^{T X}$. Following [65, (3.10)], let $h(x)$ be the function on $U$ defined by

$$
h(x)=(\operatorname{vol}(G x))^{1 / 2} .
$$

Then $h$ descends to a function on $B$.

Let $\mathrm{pr}_{1}$ and $\mathrm{pr}_{2}$ be the projections from $X \times X$ onto the first and the second factor $X$ respectively. Then we can view $P_{p}^{G}\left(x, x^{\prime}\right)\left(x, x^{\prime} \in U\right)$ as a smooth section of $\operatorname{pr}_{1}^{*}\left(E_{p}\right)_{B} \otimes \operatorname{pr}_{2}^{*}\left(E_{p}^{*}\right)_{B}$ on $B \times B$.

We introduce the following coordinates: for any $x_{0} \in X_{G}, Z \in T_{x_{0}} B$, we write $Z=Z^{0}+Z^{\perp}$, with $Z^{0} \in T_{x_{0}} X_{G}, Z^{\perp} \in N_{G, x_{0}}$, where $N_{G}$ is the normal bundle of $X_{G}$ in $B$. For $\varepsilon_{0}>0$ small enough, we identify $Z \in T_{x_{0}} B,|Z|<\varepsilon_{0}$ with $\exp _{\exp _{x_{0}}^{B}{ }_{X_{G}}^{\left.X_{G}\right)}}\left(Z^{\perp}\right) \in B$, here we still denote by $Z^{\perp} \in N_{G \text {, } \exp _{x_{0}} X_{G}\left(Z^{0}\right)}$, the parallel transport of $Z^{\perp}$ along the curve $u \rightarrow \exp _{x_{0}}^{X_{G}}\left(u Z^{0}\right)$ with respect to the connection on $N_{G}$ induced by projecting the Levi-Civita connection on $T B$.

We identify $\left(E_{p}\right)_{B, Z}$ with $\left(E_{p}\right)_{B, x_{0}}$ by using parallel transport with respect to $\nabla^{\left(E_{p}\right)_{B}}$ (cf. $\S 2.1$ ) along the curve $[0,1] \ni u \rightarrow u Z$.

Let $d v_{B}, d v_{X_{G}}, d v_{N_{G}}$ be the Riemannian volume forms on $T B, T X_{G}, N_{G}$ induced by $g^{T X}$. Let $\varrho \in \mathscr{C}^{\infty}\left(\left.T B\right|_{X_{G}}, \mathbb{R}\right)$, with $\varrho=1$ on $X_{G}$, be defined by

$$
\begin{gathered}
d v_{B}\left(x_{0}, Z\right)=\varrho\left(x_{0}, Z\right) d v_{X_{G}}\left(x_{0}\right) d v_{N_{G, x_{0}}} \quad \text { for } Z \in T_{x_{0}} B, x_{0} \in X_{G} . \\
\text { For } x_{0} \in X_{G}, Z=\left(Z^{0}, Z^{\perp}\right), Z^{\prime}=\left(Z^{\prime 0}, Z^{\prime \perp}\right) \in T_{x_{0}} X_{G} \oplus N_{G, x_{0}}=T_{x_{0}} B \text {, set } \\
\mathcal{P}\left(Z, Z^{\prime}\right)=2^{\frac{n_{0}}{2}} \exp \left(-\frac{\pi}{2} \sum_{i}\left(\left|z_{i}^{0}\right|^{2}+\left|z_{i}^{\prime 0}\right|^{2}-2 z_{i}^{0} \bar{z}_{i}^{\prime 0}\right)\right) \\
\times \exp \left(-\pi\left|Z^{\perp}\right|^{2}-\pi\left|Z^{\perp}\right|^{2}\right)
\end{gathered}
$$

with $n_{0}=\operatorname{dim} G$. As in (1.16) and (2.9), $\mathcal{P}$ is the Bergman kernel of a limit operator, which itself is sum of two terms: one is defined on $T_{x_{0}} X_{G}$, and is equal $\mathscr{L}$ (cf. (1.13)); the other is defined on $N_{G, x_{0}}$, it is equal to a harmonic oscillator. This explains why we expect the $G$-invariant Bergman kernel $P_{p}^{G}\left(x, x^{\prime}\right)$ to exhibit the same sort of behavior, see (3.11).

Let $\left\{\Theta_{p}^{G}\right\}_{p \in \mathbb{N}}$ be a sequence of linear operators $\Theta_{p}^{G}: L^{2}\left(X, E_{p}\right) \longrightarrow$ $L^{2}\left(X, E_{p}\right)$ with smooth kernel $\Theta_{p}^{G}(x, y)$ with respect to $d v_{X}(y)$. We assume 
that $\Theta_{p}^{G}(x, y)$ is $G \times G$-invariant. Let $\pi_{B}: T B \times_{X_{G}} T B \rightarrow X_{G}$ be the obvious projection. Relative to our trivialization, $\Theta_{p}^{G}(x, y)$ induces a smooth section $\Theta_{p, x_{0}}^{G}\left(Z, Z^{\prime}\right)$ of $\pi_{B}^{*}\left(\operatorname{End}\left(\Lambda^{0, \bullet} \otimes E\right)_{B}\right)$ over $T B \times_{X_{G}} T B$ with $Z, Z^{\prime} \in T_{x_{0}} B$. We introduce the following notation in analogy to Notation 2.1.

Notation 3.3. We write

$$
p^{-n+\frac{n_{0}}{2}} \Theta_{p, x_{0}}^{G}\left(Z, Z^{\prime}\right) \stackrel{h}{\approx} \sum_{r=0}^{k}\left(Q_{r, x_{0}}^{G} \mathcal{P}_{x_{0}}\right)\left(\sqrt{p} Z, \sqrt{p} Z^{\prime}\right) p^{-\frac{r}{2}}+\mathcal{O}\left(p^{-\frac{k+1}{2}}\right)
$$

if there exists a family $\left\{Q_{r, x_{0}}^{G}\right\}_{0 \leqslant r \leqslant k, x_{0} \in X_{G}}$ with $Q_{r, x_{0}}^{G} \in \operatorname{End}\left(\Lambda^{0, \bullet} \otimes\right.$ $E)_{B, x_{0}}\left[Z, Z^{\prime}\right]$ smooth with respect to the parameter $x_{0} \in X_{G}$, and there exist $\left.\varepsilon^{\prime} \in\right] 0, a^{X}$ [ and $C_{0}>0$ with the following property: for any $l, m \in \mathbb{N}$, there exist $C>0, M>0$ such that for any $x_{0} \in X_{G}, Z, Z^{\prime} \in T_{x_{0}} B,|Z|,\left|Z^{\prime}\right|<\varepsilon^{\prime}$ and $p \in \mathbb{N}^{*}$, the following estimate holds:

$$
\begin{gathered}
\left(1+\sqrt{p}\left|Z^{\perp}\right|+\sqrt{p}\left|Z^{\perp \perp}\right|\right)^{m} \mid p^{-n+\frac{n_{0}}{2}} \Theta_{p, x_{0}}^{G}\left(Z, Z^{\prime}\right)\left(h \varrho^{\frac{1}{2}}\right)(Z)\left(h \varrho^{\frac{1}{2}}\right)\left(Z^{\prime}\right) \\
-\left.\sum_{r=0}^{k}\left(Q_{r, x_{0}}^{G} \mathcal{P}_{x_{0}}\right)\left(\sqrt{p} Z, \sqrt{p} Z^{\prime}\right) p^{-\frac{r}{2}}\right|_{\mathscr{C}^{l}\left(X_{G}\right)} \\
\leqslant C p^{-\frac{k+1}{2}}\left(1+\sqrt{p}\left|Z^{0}\right|+\sqrt{p}\left|Z^{\prime 0}\right|\right)^{M} \exp \left(-\sqrt{C_{0} p}\left|Z-Z^{\prime}\right|\right)+\mathscr{O}\left(p^{-\infty}\right) .
\end{gathered}
$$

Theorem 3.4 (Ma-Zhang [43, Th. 0.2]). There exists a family of polynomials $\left\{\mathcal{Q}_{r, x_{0}}\right\}_{r \in \mathbb{N}, x_{0} \in X_{G}} \in \operatorname{End}\left(\Lambda^{0, \bullet} \otimes E\right)_{B, x_{0}}\left[Z, Z^{\prime}\right]$ on $Z, Z^{\prime}$ with the same parity as $r$, such that $\mathcal{Q}_{0, x_{0}}=I_{\mathbb{C} \otimes E, G}$, and for any $k \in \mathbb{N}$ the following expansion holds in the sense of Notation 3.3,

$$
p^{-n+\frac{n_{0}}{2}} P_{p, x_{0}}^{G}\left(Z, Z^{\prime}\right) \stackrel{h}{\approx} \sum_{r=0}^{k}\left(\mathcal{Q}_{r, x_{0}} \mathcal{P}_{x_{0}}\right)\left(\sqrt{p} Z, \sqrt{p} Z^{\prime}\right) p^{-\frac{r}{2}}+\mathcal{O}\left(p^{-\frac{k+1}{2}}\right) .
$$

To read off the scalar curvature on the reduction from $P_{p}^{G}$, we define $\mathscr{I}_{p}\left(x_{0}\right) \in \operatorname{End}\left(\Lambda^{0, \bullet} \otimes E\right)_{G, x_{0}}$ for $x_{0} \in X_{G}$ by :

$$
\mathscr{I}_{p}\left(x_{0}\right)=\int_{\substack{|Z| \leqslant \varepsilon_{0}, Z \in N_{G}}}\left(\varrho h^{2}\right)\left(x_{0}, Z\right) P_{p}^{G}\left(\left(x_{0}, Z\right),\left(x_{0}, Z\right)\right) d v_{N_{G}}(Z) .
$$

By (3.4), (3.5), $\mathscr{I}_{p}\left(x_{0}\right)$ does not depend on $\varepsilon_{0}$ modulo $\mathscr{O}\left(p^{-\infty}\right)$, and

$$
\operatorname{dim} \operatorname{Ker}\left(D_{G, p}\right)=\int_{X_{G}} \operatorname{Tr}\left[\mathscr{I}_{p}\left(x_{0}\right)\right] d v_{X_{G}}\left(x_{0}\right)+\mathscr{O}\left(p^{-\infty}\right) .
$$

From Theorem 3.4, we infer the existence of $\Phi_{r} \in \mathscr{C}^{\infty}\left(X_{G}, \operatorname{End}\left(\Lambda^{0, \bullet} \otimes E\right)_{G}\right)$, and $\Phi_{0}=I_{\mathbb{C} \otimes E, G}$, with the property that for all $k, m \in \mathbb{N}$, there exists $C_{k, m}>0$ 
such that for all $p \in \mathbb{N}^{*}$,

$$
\left|p^{-n+n_{0}} \mathscr{I}_{p}\left(x_{0}\right)-\sum_{r=0}^{k} \Phi_{r}\left(x_{0}\right) p^{-r}\right|_{\mathscr{C} m\left(X_{G}\right)} \leqslant C_{k, m} p^{-k-1}
$$

Using Theorems 3.2, 3.4, and the same argument as in Remark 2.5, we see that the analogue of Theorems $3.2,3.4$ still holds for the kernel $T_{f, p}^{G}\left(x, x^{\prime}\right)$ of the operator $T_{f, p}^{G}:=P_{p}^{G} f P_{p}^{G}$, for $f \in \mathscr{C}^{\infty}(X, \operatorname{End}(E))$.

Theorem 3.5 (Ma-Zhang [43, p. 86-88]). Let $f \in \mathscr{C}^{\infty}(X, \operatorname{End}(E))$. For any open $G$-neighborhood $U$ of $\mu^{-1}(0), \varepsilon_{0}>0$, we have

$$
T_{f, p}^{G}\left(x, x^{\prime}\right)=\mathscr{O}\left(p^{-\infty}\right) \text { if }\left(x, x^{\prime}\right) \notin U \times U \text { or if } d^{X}\left(G x, x^{\prime}\right) \geqslant \varepsilon_{0} .
$$

Moreover, there exists a family $\left\{\mathcal{Q}_{r, x_{0}}^{G}(f)\right\}_{r \in \mathbb{N}, x_{0} \in X_{G}} \in \operatorname{End}\left(\Lambda^{0, \bullet} \otimes\right.$ $E)_{B, x_{0}}\left[Z, Z^{\prime}\right]$ of polynomials in $Z, Z^{\prime}$ with the same parity as $r$ such that for any $k \in \mathbb{N}$, we have in the sense of Notation 3.3,

$$
p^{-n+\frac{n_{0}}{2}} T_{f, p, x_{0}}^{G}\left(Z, Z^{\prime}\right) \stackrel{h}{\approx} \sum_{r=0}^{k}\left(\mathcal{Q}_{r, x_{0}}^{G}(f) \mathcal{P}_{x_{0}}\right)\left(\sqrt{p} Z, \sqrt{p} Z^{\prime}\right) p^{-\frac{r}{2}}+\mathcal{O}\left(p^{-\frac{k+1}{2}}\right) .
$$

Moreover, $\mathcal{Q}_{0, x_{0}}^{G}(f)=f^{G}\left(x_{0}\right) I_{\mathbb{C} \otimes E, G}$, where $f^{G}$ is the $G$-invariant component of $f$.

Since $\operatorname{Tr}\left[T_{f, p}^{G}\right]=\int_{X} \operatorname{Tr}\left[T_{f, p}^{G}(x, x)\right] d v_{X}(x)$, we deduce from Theorem 3.5 that there exists a sequence $B_{r, f}$ with $B_{0, f}=\int_{X_{G}} \operatorname{Tr}\left[f^{G}\left(x_{0}\right)\right] d v_{X_{G}}\left(x_{0}\right)$ and for any $k \in \mathbb{N}$,

$$
p^{-n+n_{0}} \operatorname{Tr}\left[T_{f, p}^{G}\right]=\sum_{r=0}^{k} B_{r, f} p^{-r}+\mathscr{O}\left(p^{-k-1}\right) .
$$

Note that in $[43, \S 4.1, \S 4.5]$ the case where 0 is a regular value of $\mu$ (so that $X_{G}$ is an orbifold) is treated in detail. In [43, §4.2], it is shown by a shifting trick that Theorems 3.2 and 3.4 imply the expansion of the kernel of the orthogonal projection $P_{p}^{V_{\gamma}^{G}}$ from $\Omega^{0, \bullet}\left(X, L^{p} \otimes E\right)$ onto the $V_{\gamma}^{G}$-component of $\operatorname{Ker}\left(D_{p}\right)$ for any $\gamma \in \Lambda_{+}^{*}$.

3.3. The Kähler case. In this subsection, as in Section 2.3, we assume that $(X, \omega, J)$ is a compact Kähler manifold carrying a holomorphic Hermitian line bundle $\left(L, h^{L}\right)$ and a holomorphic Hermitian vector bundle $\left(E, h^{E}\right)$ and moreover $\omega=\frac{\sqrt{-1}}{2 \pi} R^{L}$ is the Kähler form of $\left(X, g^{T X}\right)$. We assume also that the $G$-action on $X, L, E$ is holomorphic, and preserves the metrics.

By $(2.19)$, we see as in Section 2.3 that the $G$-invariant Bergman projection $P_{p}^{G}$ reduces to a projection from $\mathscr{C}^{\infty}\left(X, L^{p} \otimes E\right)$ onto $H^{0}\left(X, L^{p} \otimes E\right)^{G}$, and 
the Toeplitz operator $\left\{T_{f, p}^{G}\right\}$ reduces to a sequence of linear operators acting on $\mathscr{C}^{\infty}\left(X, L^{p} \otimes E\right)$. In particular, $\mathcal{Q}_{r, x_{0}}, \mathscr{I}_{p}\left(x_{0}\right), \Phi_{r}\left(x_{0}\right), \mathcal{Q}_{r, x_{0}}^{G}(f)$ in (3.11), (3.14) and (3.16) take values in $\operatorname{End}\left(E_{G}\right)_{x_{0}}$.

Let $\widetilde{h}$ be the restriction of $h$ on $X_{G}$. Let $r^{X_{G}}$ be the scalar curvature on $\left(X_{G}, \omega_{G}, J_{G}\right)$, and $\Delta_{X_{G}}$ be the positive Laplacian on $X_{G}$. Let $\left\{w_{j}^{0}\right\}$ be an orthonormal frame of $T^{(1,0)} X_{G}$. The following result generalizes formula (2.13) for the coefficient $\boldsymbol{b}_{1}$ of the expansion (2.7).

Theorem 3.6 (Ma-Zhang [43, Th. 0.6]). The coefficients $\Phi_{0}$ and $\Phi_{1}$ from (3.14) are given by,

$\Phi_{0}=\operatorname{Id}_{E_{G}}, \quad \Phi_{1}\left(x_{0}\right)=\frac{1}{8 \pi} r_{x_{0}}^{X_{G}}+\frac{3}{4 \pi} \Delta_{X_{G}} \log \widetilde{h}+\frac{1}{2 \pi} \sum_{j} R_{x_{0}}^{E_{G}}\left(w_{j}^{0}, \bar{w}_{j}^{0}\right)$.

We discuss now the metric aspect of quantization. Let $i: \mu^{-1}(0) \hookrightarrow X$ be the natural injection. Let $\pi_{G}: \mathscr{C}^{\infty}\left(\mu^{-1}(0), L^{p} \otimes E\right)^{G} \rightarrow \mathscr{C}^{\infty}\left(X_{G}, L_{G}^{p} \otimes E_{G}\right)$ be the natural identification. By a result of Zhang [73, Th. 1.1 and Prop. 1.2], for $p \gg 1$, the map $\pi_{G} \circ i^{*}: \mathscr{C}^{\infty}\left(X, L^{p} \otimes E\right)^{G} \rightarrow \mathscr{C}^{\infty}\left(X_{G}, L_{G}^{p} \otimes E_{G}\right)$ induces a natural isomorphism

$$
\sigma_{p}=\pi_{G} \circ i^{*}: H^{0}\left(X, L^{p} \otimes E\right)^{G} \rightarrow H^{0}\left(X_{G}, L_{G}^{p} \otimes E_{G}\right) .
$$

(When $E=\mathbb{C}$, this result was first proved in [29, Th. 3.8] for $p \geqslant 1$ ). We denote by $\langle\cdot, \cdot\rangle$ the $L^{2}$-Hermitian products on these spaces. A corollary of Theorem 3.5 is as follows.

Theorem 3.7 (Ma-Zhang [43, Th.4.8]). Set $\sigma_{p}^{G}=\sigma_{p} \circ P_{p}^{G}$ and let $\sigma_{p}^{G *}$ be the adjoint of $\sigma_{p}^{G}$. Then $\mathcal{T}_{f, p}=p^{-\frac{n_{0}}{2}} \sigma_{p}^{G} f \sigma_{p}^{G *} \in \operatorname{End}\left(H^{0}\left(X_{G}, L_{G}^{p} \otimes\right.\right.$ $\left.E_{G}\right)$ ) is a Toeplitz operator with principal symbol $2^{\frac{n_{0}}{2}} f^{G} / \widetilde{h}^{2}$, for any $f \in$ $\mathscr{C}^{\infty}(X, \operatorname{End}(E))$.

The natural Hermitian product $\langle\cdot, \cdot\rangle_{\widetilde{h}}$ on $\mathscr{C}^{\infty}\left(X_{G}, L_{G}^{p} \otimes E_{G}\right)$ is given by

$$
\left\langle s_{1}, s_{2}\right\rangle_{\widetilde{h}}=\int_{X_{G}}\left\langle s_{1}, s_{2}\right\rangle\left(x_{0}\right) \widetilde{h}^{2}\left(x_{0}\right) d v_{X_{G}}\left(x_{0}\right) .
$$

Theorem 3.8 (Ma-Zhang [43, Th. 0.10]). The isomorphism $(2 p)^{-\frac{n_{0}}{4}} \sigma_{p}$ is an asymptotic isometry from $\left(H^{0}\left(X, L^{p} \otimes E\right)^{G},\langle\cdot, \cdot\rangle\right)$ onto $\left(H^{0}\left(X_{G}, L_{G}^{p} \otimes\right.\right.$ $\left.\left.E_{G}\right),\langle\cdot, \cdot\rangle_{\widetilde{h}}\right)$, i.e., if $\left\{s_{i}^{p}\right\}_{i=1}^{d_{p}}$ is an orthonormal basis of $\left(H^{0}\left(X, L^{p} \otimes E\right)^{G},\langle\cdot, \cdot\rangle\right)$, then

$$
(2 p)^{-\frac{n_{0}}{2}}\left\langle\sigma_{p} s_{i}^{p}, \sigma_{p} s_{j}^{p}\right\rangle_{\widetilde{h}}=\delta_{i j}+\mathscr{O}\left(p^{-1}\right) .
$$

In [43, Remark 0.11], we find a natural symplectic extension of Theorem 3.8 . 
When $E=\mathbb{C}$ and $G$ is a torus, Charles [19] first showed that $\mathcal{T}_{f, p}$ in Theorem 3.7 is a Toeplitz operator, and obtained (3.21).

Assume that $E=\mathbb{C}$. Then $P_{p}^{G}\left(x_{0}, x_{0}\right)$ becomes a positive function. By setting $Z=Z^{\prime}=0$ in (3.11), we get the following expansion on $X_{G}$ for any $k$,

$$
p^{-n+\frac{n_{0}}{2}} h^{2}\left(x_{0}\right) P_{p}^{G}\left(x_{0}, x_{0}\right)=\sum_{r=0}^{k} c_{r}\left(x_{0}\right) p^{-r}+\mathscr{O}\left(p^{-k-1}\right), \quad c_{0}\left(x_{0}\right)=2^{n_{0} / 2} .
$$

Paoletti [50, Th. 1], [51, Th. 1] had obtained the expansion (3.22), but he claimed that $c_{0}\left(x_{0}\right)=1$. After our preprint [43] was posted, Hall-Kirwin [30], Paoletti [52], [53] and Burns-Guillemin-Wang [16] have established related results.

\section{Noncompact Case: Vergne's Conjecture}

In this section, we use the same notation and assumptions as in Sections 1, 3.1 , except that we assume now that $X$ is noncompact. One asks naturally the following question: what is the quantization formula in this situation?

When $\left(X, g^{T X}\right)$ is a complete Riemannian manifold, it is shown in [38, §3.5], $[40, \S 5],[41, \S 6.1, \S 7.5],[43, \S 4.6]$ that under natural (positivity) conditions on $R^{L}, R^{E}$, the asymptotic expansion of the Bergman kernel holds. However, in this section, we do not assume $\left(X, g^{T X}\right)$ to be complete.

In Section 4.1, the quantization formula is explained for the model example $\mathbb{C}^{n}$. In Section 4.2, we review briefly our solution with Zhang of Vergne's conjecture: "quantization commutes with reduction" in the noncompact setting.

4.1. Quantization formula on $\mathbb{C}^{n}$. We continue the discussion of Section 1.2. Let's assume now that $a_{j}=2 \pi$ for $j=1, \cdots, n$. Then $\left(L, h^{L}, \nabla^{L}\right)$ is a prequantum line bundle on $\left(\mathbb{C}^{n}, \omega=\frac{\sqrt{-1}}{2} \sum_{j} d z_{j} \wedge d \bar{z}_{j}\right)$.

Let $T^{n}$ be the $n$-dimensional torus with Lie algebra $\mathfrak{t}^{n}$. We define a holomorphic action of $T^{n}$ on $\mathbb{C}^{n}$ by $e^{i \theta} \cdot z=\left(e^{i \theta_{1}} z_{1}, \cdots, e^{i \theta_{n}} z_{n}\right)$, with $\theta=\left(\theta_{1}, \cdots, \theta_{n}\right) \in$ $\mathbb{R}^{n}$ and $e^{i \theta}=\left(e^{i \theta_{1}}, \cdots, e^{i \theta_{n}}\right) \in T^{n}$. For $\lambda=\left(\lambda_{1}, \cdots, \lambda_{n}\right) \in \mathbb{Z}^{n}$, we define a holomorphic $T^{n}$-action on $L$ by $e^{i \theta} \cdot \mathbf{1}=e^{i \theta \cdot \lambda} \mathbf{1}$ with $\theta \cdot \lambda=\sum_{j} \theta_{j} \lambda_{j}$. Then the associated moment map $\mu: \mathbb{C}^{n} \rightarrow \mathbb{R}^{n *}$ (cf. (3.2)) is given by

$$
\mu(z)=\frac{1}{2}\left(\left|z_{1}\right|^{2}, \cdots,\left|z_{n}\right|^{2}\right)+\lambda .
$$

Given $\left\{u_{i}\right\}_{i=1}^{n} \subset \mathbb{Z}^{m}$, the Delzant polytope $\Delta \subset \mathbb{R}^{m *}[2$, §VII. 1.c., 2.a.] is defined by

$$
\Delta=\left\{x \in \mathbb{R}^{m *}:\left(u_{i}, x\right) \geqslant \lambda_{i} \text { for } 1 \leqslant i \leqslant n\right\},
$$

if the vertices have integer coordinates and each vertex $q$ has exactly $m$-edges, and the $u_{i}$ such that $\left(u_{i}, q\right)=\lambda_{i}$ form a basis of $\mathbb{Z}^{m}$. 
Let $\jmath: \mathbb{R}^{n} \rightarrow \mathbb{R}^{m}$ be the linear map defined by $\jmath\left(e_{i}\right)=u_{i}$ with $\left\{e_{i}\right\}$ the canonical basis of $\mathbb{R}^{n}$. Let $N=\operatorname{Ker}(\jmath) /\left(\operatorname{Ker}(\jmath) \cap(2 \pi \mathbb{Z})^{n}\right) \subset \mathbb{R}^{n} /(2 \pi \mathbb{Z})^{n} \simeq T^{n}$, so that $N$ is a $(n-m)$-dimensional torus with Lie algebra $\mathfrak{n} \stackrel{\imath}{\hookrightarrow} \mathbb{R}^{n} \simeq \mathfrak{t}^{n}$. Thus we have the exact sequence: $0 \rightarrow \mathbb{R}^{m *} \stackrel{J^{*}}{\longrightarrow} \mathbb{R}^{n *} \stackrel{\imath^{*}}{\longrightarrow} \mathfrak{n}^{*} \rightarrow 0$.

Now $N$ acts naturally on $\mathbb{C}^{n}$ and $L$, the associated moment map is $\Phi=$ $\imath^{*} \circ \mu: \mathbb{C}^{n} \rightarrow \mathfrak{n}^{*}$. Its symplectic reduction $X_{\Delta}=\Phi^{-1}(0) / N$ is a $m$-dimensional compact Kähler manifold, and $L$ descends naturally to a positive holomorphic line bundle $L_{\Delta}$ on $X_{\Delta}$. Then $X_{\Delta}$ is the toric variety associated to the Delzant polytope $\Delta$.

Observe that if $N$ acts trivially on a holomorphic section $z^{\alpha} \mathbf{1}$ of $L$ for some $\alpha \in \mathbb{N}^{n}$, then $z^{\alpha} \mathbf{1}$ descends to a holomorphic section of $L_{\Delta}$ on $X_{\Delta}$.

For $e^{i \theta} \in T^{n}$, we have $e^{i \theta} \cdot z^{\alpha} \mathbf{1}=e^{i \theta \cdot(\alpha+\lambda)} z^{\alpha} \mathbf{1}$. Thus $N$ acts trivially on the holomorphic section $z^{\alpha} \mathbf{1}$ if and only if $\imath^{*}(\alpha+\lambda)=0$, and this is equivalent to the existence of a $\nu \in \mathbb{R}^{m *}$ such that $\alpha_{i}+\lambda_{i}=\left(\nu, u_{i}\right)$, i.e., $\nu \in \Delta \cap \mathbb{Z}^{m}$ and $\alpha_{i}+\lambda_{i}=\left(\nu, u_{i}\right)$.

For $\nu \in \Delta \cap \mathbb{Z}^{m}$, we denote by $s_{\nu}$ the holomorphic section of $L_{\Delta}$ on $X_{\Delta}$ induced by $z^{\alpha} \mathbf{1}$, where $\alpha_{i}=\left(\nu, u_{i}\right)-\lambda_{i}$.

Theorem $4.1([28, \S 3.5])$. The cohomology of $L_{\Delta}$ on $X_{\Delta}$ is given by

$$
H^{0}\left(X_{\Delta}, L_{\Delta}\right)=\bigoplus_{\nu \in \Delta \cap \mathbb{Z}^{m}} \mathbb{C} s_{\nu}, \quad H^{j}\left(X_{\Delta}, L_{\Delta}\right)=0 \text { if } j>0 .
$$

By Theorem 1.5, we see that the kernel of $D^{L}$ on the noncompact space $\mathbb{C}^{n}$ is an infinite dimensional vector space. Moreover, by the discussion after (1.13) we deduce that all higher $L^{2}$ cohomology groups of $\mathbb{C}^{n}$ with values in $L$ vanish. Theorem 4.1 implies that "quantization commutes with reduction" still holds. Note that the moment map $\Phi=\imath^{*} \circ \mu$ is proper here.

Example 4.2. Set $m=n-1, u_{i}=e_{i}$ for $i \leqslant m, u_{n}=-(1, \cdots, 1)=-\sum_{i=1}^{m} e_{i}$, $\lambda=(0, \cdots, 0,-1)$. Then $\operatorname{Ker}(\jmath)=\mathbb{R}(1, \cdots, 1), \Phi(z)=\frac{1}{2} \sum_{i=1}^{n}\left|z_{i}\right|^{2}-1$. In this case, $\left(X_{\Delta}, L_{\Delta}\right) \simeq\left(\mathbb{C P}^{n-1}, \mathcal{O}(1)\right)$ with $\mathcal{O}(1)$ the hyperplane line bundle on $\mathbb{C P}^{n-1}$.

4.2. Vergne's conjecture. Recall that $(X, \omega, J)$ is a noncompact symplectic manifold with the prequantum line bundle $\left(L, h^{L}, \nabla^{L}\right)$, and $g^{T X}$ is a $J$-invariant Riemannian metric on $X$. Let $\tau: T X \rightarrow X$ be the natural projection. Following [1, p. 7] (cf. [54, §3]), set $T_{G} X=$ $\left\{(x, v) \in T_{x} X:\left\langle v, K^{X}(x)\right\rangle=0\right.$ for all $\left.K \in \mathfrak{g}\right\}$.

Then the quantization space $Q(L)=\operatorname{Ind}\left(D^{L}\right)$ of $L$ is not well defined, since usually $D^{L}$ is not a Fredholm operator, and we need to make precise the self-adjoint extension of $\left.D^{L}\right|_{\Omega_{0}^{0, \bullet}(X, L)}$, where $\Omega_{0}^{0, \bullet}(X, L)$ denotes the space of sections with compact support.

We suppose that the moment map $\mu: X \rightarrow \mathfrak{g}^{*}$ is proper. Then the right hand side of (3.3) is well defined. 
We identify $\mathfrak{g}$ with $\mathfrak{g}^{*}$ by using an $\operatorname{Ad}_{G}$-invariant metric on $\mathfrak{g}$. Let $\mu^{X}(x):=$ $(\mu(x))^{X}(x)(x \in X)^{1}$ be the vector field induced by $\mu: X \rightarrow \mathfrak{g}$.

We suppose for the moment that $\left\{x \in X: \mu^{X}(x)=0\right\}$ is compact.

Recall that $\mathbf{c}(\cdot)$ is the Clifford action defined in (1.1). For $x \in X, \xi \in T_{x} X$, set $^{2}$

$$
\begin{aligned}
\sigma_{L, \mu}^{X}(x, \xi)=\tau^{*} & \left.\left(\sqrt{-1} \mathbf{c}\left(\xi+\mu^{X}\right) \otimes \operatorname{Id}_{L}\right)\right|_{(x, \xi)} \\
& : \tau^{*}\left(\Lambda^{\text {even }}\left(T^{*(0,1)} X\right) \otimes L\right) \rightarrow \tau^{*}\left(\Lambda^{\text {odd }}\left(T^{*(0,1)} X\right) \otimes L\right)
\end{aligned}
$$

Then $\sigma_{L, \mu}^{X}$ is a transversally elliptic symbol on $T_{G} X$ in the sense of Atiyah $[1, \S 1, \S 3]$ and Paradan $[54, \S 3],[55, \S 3]$, which determines a transversal index Ind $\left(\sigma_{L, \mu}^{X}\right)$ in the formal representation ring $R[G]$ of $G$,

$$
\operatorname{Ind}\left(\sigma_{L, \mu}^{X}\right)=\bigoplus_{\gamma \in \Lambda_{+}^{*}} \operatorname{Ind}_{\gamma}\left(\sigma_{L, \mu}^{X}\right) \cdot V_{\gamma}^{G} \in R[G]
$$

The index Ind $\left(\sigma_{L, \mu}^{X}\right)$ does not depend on $g^{T X}, h^{L}, \nabla^{L}$, and it depends only on the homotopy classes of $J, \mu^{X}$. The set $\left\{\gamma \in \Lambda_{+}^{*}: \operatorname{Ind}_{\gamma}\left(\sigma_{L, \mu}^{X}\right) \neq 0\right\}$ can be infinite. Michèle Vergne suggested to use $\operatorname{Ind}_{\gamma}\left(\sigma_{L, \mu}^{X}\right)$ to replace the left hand side of (3.3).

Vergne's conjecture (ICM 2006 plenary lecture $[69, \S 4.3]):$ If $\mu: X \rightarrow \mathfrak{g}^{*}$ is proper and if $\left\{x \in X: \mu^{X}(x)=0\right\}$ is compact, then for any $\gamma \in \Lambda_{+}^{*}$,

$$
\operatorname{Ind}_{\gamma}\left(\sigma_{L, \mu}^{X}\right)=Q\left(L_{\gamma}\right)
$$

Special cases of this conjecture, related to the discrete series of semi-simple Lie groups, have been proved by Paradan [55], [57].

For $a>0$, set $X_{a}=\left\{x \in X:|\mu|^{2}(x) \leqslant a\right\}$. If $a$ is a regular value of $|\mu|^{2}$, then $X_{a}$ is a compact manifold with boundary $\partial X_{a}$, and $\mu^{X}$ is nowhere zero on $\partial X_{a}$. Thus $\sigma_{L, \mu}^{X_{a}}$ is a transversally elliptic symbol on $X_{a}$.

Theorem 4.3 (Quantization commutes with reduction, Ma-Zhang [45, Th. 0.2,0.3]). Suppose that $\mu: X \rightarrow \mathfrak{g}^{*}$ is proper. For any $\gamma \in \Lambda_{+}^{*}$, there exists $a_{\gamma}>0$ such that the function $a \mapsto \operatorname{Ind}_{\gamma}\left(\sigma_{L, \mu}^{X_{a}}\right)$ is constant on $\{a>$ $a_{\gamma}$ : a is regular value of $\left.|\mu|^{2}\right\}$. Denote by $Q(L)^{\gamma}$ this constant. Then for any $\gamma \in \Lambda_{+}^{*}$, we have

$$
Q(L)^{\gamma}=Q\left(L_{\gamma}\right)
$$

\footnotetext{
${ }^{1}$ The vector field $\mu^{X}$ is also called Kirwan vector field in view of [32].

${ }^{2}$ The symbol $\sigma_{L, \mu}^{X}$ is the (semi-classical) symbol of Tian-Zhang's [65] deformed Dirac operator (4.8) in their approach to the Guillemin-Sternberg geometric quantization conjecture.
} 
If $\left\{x \in X: \mu^{X}(x)=0\right\}$ is compact, then $Q(L)^{\gamma}=\operatorname{Ind}_{\gamma}\left(\sigma_{L, \mu}^{X}\right)$. Therefore Theorem 4.3 implies Vergne's conjecture. Note that Paradan [58] gives a new proof of Theorem 4.3 by using symplectic cuts and the wonderful compactifications of [56].

Idea of the proof. 1) Assume that $\left\{x \in X: \mu^{X}(x)=0\right\}$ is compact. For $T>0$, let $D_{T}^{L}$ be the deformed Dirac operator introduced by Tian-Zhang $[65,(1.20)]$ :

$$
D_{T}^{L}=D^{L}+\sqrt{-1} T \mathbf{c}\left(\mu^{X}\right): \Omega^{0, \bullet}(X, L) \rightarrow \Omega^{0, \bullet}(X, L) .
$$

A first step is to interpret the transversal index as the Atiyah-Patodi-Singer index of $D_{T}^{L}$ for a manifold with boundary defined as in [66]. The proof uses Braverman's $L^{2}$-interpretation of the transversal index $[15, \S 5]$. The proof of (4.7) for $\gamma=0$ is then easy.

2) A second key result is as follows. Let $\left(N, \omega^{N}, J^{N}\right)$ be a compact symplectic manifold with a prequantum line bundle $\left(F, h^{F}, \nabla^{F}\right)$ (see Section 1.1). We suppose that $G$ acts on $N$ and the action lifts to $F$ as above with the associated moment map $\eta: N \rightarrow \mathfrak{g}^{*}$, etc. For $\gamma \in \Lambda_{+}^{*}$, set $Q(F)^{-\gamma}=\operatorname{dim}_{\operatorname{Hom}_{G}}\left(\left(V_{\gamma}^{G}\right)^{*}, Q(F)\right)$, where $\operatorname{Hom}_{G}$ is the linear space of $G$ homomorphisms. Let $L \otimes F$ be the obvious prequantum line bundle over $X \times N$. Then we have

$$
Q(L \otimes F)^{\gamma=0}=\sum_{\gamma \in \Lambda_{+}^{*}} Q(L)^{\gamma} \cdot Q(F)^{-\gamma} .
$$

The proof of Theorem 4.3 is obtained in [45] by combining these two arguments.

\section{References}

[1] M. F. Atiyah, Elliptic operators and compact groups, Lecture Notes in Mathematics, Vol. 401, Springer-Verlag, Berlin, 1974.

[2] M. Audin, Torus actions on symplectic manifolds. Second revised edition. Progress in Mathematics, 93. Birkhäuser Verlag, Basel, 2004. viii+325 pp.

[3] N. Berline, E. Getzler, and M. Vergne, Heat kernels and Dirac operators, Grundl. Math. Wiss. Band 298, Springer-Verlag, Berlin, 1992.

[4] R. Berman, B. Berndtsson, and J. Sjöstrand, A direct approach to Bergman kernel asymptotics for positive line bundles, Ark. Mat. 46 (2008), 197-217.

[5] O. Biquard, Métriques kählériennes à courbure scalaire constante, Séminaire Bourbaki. Vol. 2004/2005. Astérisque no. 307 (2006), Exp. No. 938, vii, 1-31.

[6] J.-M. Bismut, Demailly's asymptotic inequalities: a heat equation proof, J. Funct. Anal. 72 (1987), 263-278.

[7] J.-M. Bismut and G. Lebeau, Complex immersions and Quillen metrics, Inst. Hautes Études Sci. Publ. Math. (1991), no. 74, ii+298 pp. 
[8] J.-M. Bismut and É. Vasserot, The asymptotics of the Ray-Singer analytic torsion associated with high powers of a positive line bundle, Comm. Math. Phys. 125 (1989), no. 2, 355-367.

[9] M. Bordemann, E. Meinrenken and M. Schlichenmaier, Toeplitz quantization of Kähler manifolds and $\mathrm{gl}(N), N \rightarrow \infty$ limits, Comm. Math. Phys. 165 (1994), 281-296.

[10] D. Borthwick and A. Uribe, Almost complex structures and geometric quantization, Math. Res. Lett. 3 (1996), no. 6, 845-861. Erratum: 5 (1998), 211-212.

[11] Th. Bouche, Convergence de la métrique de Fubini-Study d'un fibré linéare positif, Ann. Inst. Fourier (Grenoble) 40 (1990), 117-130.

[12] L. Boutet de Monvel and V. Guillemin, The spectral theory of Toeplitz operators, Annals of Mathematics Studies, vol. 99, Princeton University Press, 1981.

[13] L. Boutet de Monvel and J. Sjöstrand, Sur la singularité des noyaux de Bergman et de Szegö, Soc. Math. France, Paris, 1976, pp. 123-164. Astérisque, No. 34-35.

[14] M. Braverman, Vanishing theorems on covering manifolds, Contemp. Math., 213 (1999), 1-23.

[15] M. Braverman, Index theorem for equivariant Dirac operators on non-compact manifolds, K-Theory 27 (2002), no. 1, 61-101.

[16] D. Burns, V. Guillemin, Z. Wang, Stability Functions, arXiv:0804.3225, Geom. Funct. Anal.19 (2010), 1258-1295.

[17] D. Catlin, The Bergman kernel and a theorem of Tian, Analysis and geometry in several complex variables (Katata, 1997), Trends Math., Birkhäuser Boston, 1999, 1-23.

[18] L. Charles, Berezin-Toeplitz operators, a semi-classical approach, Comm. Math. Phys. 239 (2003), 1-28.

[19] L. Charles, Toeplitz operators and hamiltonian torus action, J. Funct. Anal. 236 (2006), 299-350.

[20] X. Dai, K. Liu, and X. Ma, On the asymptotic expansion of Bergman kernel, J. Differential Geom. 72 (2006), 1-41; announced in C. R. Math. Acad. Sci. Paris 339 (2004), no. 3, 193-198.

[21] J.-P. Demailly, Champs magnétiques et inegalités de Morse pour la $d^{\prime \prime}-$ cohomologie, Ann. Inst. Fourier (Grenoble) 35 (1985), 189-229.

[22] S. K. Donaldson, Remarks on gauge theory, complex geometry and 4-manifold topology, Fields Medallists' lectures, 384-403, World Sci. Ser. 20th Century Math., 5, World Sci. Publishing, River Edge, NJ, 1997.

[23] S. K. Donaldson, Scalar curvature and projective embeddings. I, J. Differential Geom. 59 (2001), no. 3, 479-522.

[24] B. Fedosov, Deformation quantization and index theory. Mathematical Topics. 9. Berlin: Akademie Verlag. 1996, 325 pp.

[25] C. Fefferman, The Bergman kernel and biholomorphic mappings of pseudoconvex domains, Invent. Math. 26 (1974), 1-65. 
[26] J. Fine, Calabi flow and projective embeddings, with an appendix by K. Liu and X. Ma, Asymptotic of the operators $Q_{k}$, arXiv: 0811.0155. J. Differential Geom. to appear.

[27] J. Fine, to appear.

[28] W. Fulton, Introduction to toric varieties, Annals of Mathematics Studies, 131. Princeton University Press, Princeton, NJ, 1993. xii+157 pp.

[29] V. Guillemin and S. Sternberg, Geometric quantization and multiplicities of group representations, Invent. Math. 67 (1982), no. 3, 515-538.

[30] B. Hall, W. Kirwin, Unitarity in "quantization commutes with reduction", arXiv:math/0610005, Comm. Math. Phys. 275 (2007), $401-442$.

[31] A. V. Karabegov and M. Schlichenmaier, Identification of Berezin-Toeplitz deformation quantization, J. Reine Angew. Math. 540 (2001), 49-76.

[32] F. C. Kirwan, Cohomology of quotients in symplectic and algebraic geometry. Mathematical Notes, 31. Princeton University Press, Princeton, NJ, 1984. i+211 pp.

[33] B. Kostant, Quantization and unitary representations. Lect. Notes in Math. 170 (1970), 87-207.

[34] E. Lerman, Symplectic cuts, Math. Res. Lett. 2 (1995), 247-258.

[35] K. Liu and X. Ma, A remark on 'some numerical results in complex differential geometry', Math. Res. Lett. 14 (2007), no. 2, 165-171.

[36] Z. Lu, On the lower order terms of the asymptotic expansion of Tian-YauZelditch, Amer. J. Math. 122 (2000), no. 2, 235-273.

[37] X. Ma and G. Marinescu, The $\operatorname{Spin}^{c}$ Dirac operator on high tensor powers of a line bundle, Math. Z. 240 (2002), no. 3, 651-664.

[38] X. Ma and G. Marinescu, Generalized Bergman kernels on symplectic manifolds, Adv. Math. 217 (2008), no. 4, 1756-1815; announced in C. R. Math. Acad. Sci. Paris 339 (2004), no. 7, 493-498.

[39] X. Ma and G. Marinescu, The first coefficients of the asymptotic expansion of the Bergman kernel of the spin ${ }^{c}$ Dirac operator, Internat. J. Math. 17 (2006), 737-759.

[40] X. Ma and G. Marinescu, Toeplitz operators on symplectic manifolds, J. Geom. Anal. 18 (2008), 565-611.

[41] X. Ma and G. Marinescu, Holomorphic Morse inequalities and Bergman kernels, Progress in Mathematics, vol. 254, Birkhäuser Boston Inc., 2007, 422pp.

[42] X. Ma and G. Marinescu, Berezin-Toeplitz quantization of Kähler manifolds, Preprint 2010.

[43] X. Ma and W. Zhang, Bergman kernels and symplectic reduction, arXiv:math.DG/0607404, Astérisque 318 (2008), 154 pp; announced in C. R. Math. Acad. Sci. Paris 341 (2005), 297-302; announced also in Toeplitz quantization and symplectic reduction, Differential Geometry and Physics. Eds. M.-L. Ge and W. Zhang, Nankai Tracts in Mathematics Vol. 10, World Scientific, (2006), 343-349. 
[44] X. Ma and W. Zhang, Superconnection and family Bergman kernels, C. R. Math. Acad. Sci. Paris 344 (2007), 41-44.

[45] X. Ma and W. Zhang, Geometric quantization for proper moment maps, C. R. Math. Acad. Sci. Paris 347 (2009), 389-394. Full version: arXiv: 0812.3989.

[46] V. Mathai and W. Zhang, Geometric quantization for proper actions, with an appendix by U. Bunke, arXiv:0806.3138. Adv. Math. to appear.

[47] E. Meinrenken, On Riemann-Roch formulas for multiplicities, J. Amer. Math. Soc. 9 (1996), no. 2, 373-389.

[48] E. Meinrenken, Symplectic surgery and the Spin ${ }^{c}$-Dirac operator, Adv. Math. 134 (1998), no. 2, 240-277.

[49] E. Meinrenken and R. Sjamaar, Singular reduction and quantization. Topology 38 (1999), 699-762.

[50] R. Paoletti, Moment maps and equivariant Szegö kernels, J. Symplectic Geom. 2 (2003), 133-175.

[51] R. Paoletti, The Szegö kernel of a symplectic quotient, Adv. Math. 197 (2005), 523-553.

[52] R. Paoletti, Scaling limits for equivariant Szego kernels, arXiv:math/0612547, J. Symplectic Geom. 6 (2008), 9-32.

[53] R. Paoletti, Szego kernels, Toeplitz operators, and equivariant fixed point formulae, arXiv:0707.1375, J. Anal. Math. 106 (2008), 209-236.

[54] P.-É. Paradan, Localization of the Riemann-Roch character. J. Funct. Anal. 187 (2001), no. 2, 442-509.

[55] P.-É. Paradan, $\operatorname{Spin}^{c}$-quantization and the $K$-multiplicities of the discrete series. Ann. Sci. Ecole Norm. Sup. (4) 36 (2003), no. 5, 805-845.

[56] P.-É. Paradan, Formal geometric quantization, Ann. Inst. Fourier. 59 (2009), 199-238.

[57] P.-É. Paradan, Multiplicities of the discrete series. arXiv:0812.0059, 38 pp.

[58] P.-É. Paradan, Formal geometric quantization II, arXiv:0906.4436

[59] W.-D. Ruan, Canonical coordinates and Bergman metrics, Comm. Anal. Geom. 6 (1998), no. 3, 589-631.

[60] M. Schlichenmaier, Deformation quantization of compact Kähler manifolds by Berezin-Toeplitz quantization, Conférence Moshé Flato 1999, Vol. II (Dijon), Math. Phys. Stud., vol. 22, Kluwer Acad. Publ., Dordrecht, 2000, pp. 289-306.

[61] B. Shiffman and S. Zelditch, Asymptotics of almost holomorphic sections of ample line bundles on symplectic manifolds, J. Reine Angew. Math. 544 (2002), 181222.

[62] J.-M. Souriau, Structure des systèmes dynamiques. Maîtrises de mathématiques Dunod, Paris 1970, xxxii+414 pp.

[63] C. Teleman, The quantization conjecture revisited, Ann. of Math. 152 (2000), $1-43$.

[64] G. Tian, On a set of polarized Kähler metrics on algebraic manifolds, J. Differential Geom. 32 (1990), 99-130. 
[65] Y. Tian and W. Zhang, An analytic proof of the geometric quantization conjecture of Guillemin-Sternberg, Invent. Math. 132 (1998), no. 2, 229-259.

[66] Y. Tian and W. Zhang, Quantization formula for symplectic manifolds with boundary, Geom. Funct. Anal. 9 (1999), no. 3, 596-640.

[67] M. Vergne, Multiplicities formula for geometric quantization. I, II, Duke Math. J. 82 (1996), no. 1, 143-179, 181-194.

[68] M. Vergne, Quantification géométrique et réduction symplectique. Séminaire Bourbaki, Vol. 2000/2001. Astérisque No. 282 (2002), Exp. No. 888, viii, 249-278.

[69] M. Vergne, Applications of equivariant cohomology, International Congress of Mathematicians. Vol. I, Eur. Math. Soc., Zürich, 2007, pp. 635-664.

[70] X. Wang, Canonical metrics on stable vector bundles, Comm. Anal. Geom. 13 (2005), 253-285.

[71] S. -T.Yau, Nonlinear analysis in geometry. Enseign. Math. (2) 33 (1987), 109 158.

[72] S. Zelditch, Szegö kernels and a theorem of Tian, IMRN (1998), 317-331.

[73] W. Zhang, Holomorphic quantization formula in singular reduction, Commun. Contemp. Math. 1 (1999), no. 3, 281-293. 